\title{
Cross-correlation imaging of ambient noise sources
}

\author{
Laura Ermert, ${ }^{1}$ Antonio Villaseñor ${ }^{2}$ and Andreas Fichtner ${ }^{1}$ \\ ${ }^{1}$ Department of Earth Sciences, ETH Zurich, Switzerland. E-mail: laura.ermert@erdw.ethz.ch \\ ${ }^{2}$ Institute of Earth Sciences Jaume Almera, ICTJA-CSIC, Barcelona, Spain
}

Accepted 2015 October 19. Received 2015 October 16; in original form 2015 May 6

\begin{abstract}
S UMMAR Y
We develop and apply a novel technique to image ambient seismic noise sources. It is based on measurements of cross-correlation asymmetry defined as the logarithmic energy ratio of the causal and anticausal branches of the cross-correlation function. A possible application of this technique is to account for the distribution of noise sources, a problem which currently poses obstacles to noise-based surface wave dispersion analysis and waveform inversion. The particular asymmetry measurement used is independent of absolute noise correlation amplitudes. It is shown how it can be forward-modelled and related to the noise source power-spectral density using adjoint methods. Simplified sensitivity kernels allow us to rapidly image variations in the power-spectral density of noise sources. This imaging method correctly accounts for viscoelastic attenuation and is to first order insensitive to unmodelled Earth structure. Furthermore, it operates directly on noise correlation data sets. No additional processing is required, which makes the method fast and computationally inexpensive. We apply the method to three vertical-component cross-correlation data sets of different spatial and temporal scales. Processing is deliberately minimal so as to keep observations consistent with the imaging concept. In accord with previous studies, we image seasonally changing sources of the Earth's hum in the Atlantic, Pacific and the Southern Ocean. The sources of noise in the microseismic band recorded at stations in Switzerland are predominantly located in the Atlantic and show a clear dependence on both season and frequency. Our developments are intended as a step towards full 3-D inversions for the sources of ambient noise in various frequency bands, which may ultimately lead to improvements of noise-based structural imaging.
\end{abstract}

Key words: Inverse theory; Interferometry; Surface waves and free oscillations; Wave propagation.

\section{INTRODUCTION}

Long regarded as a mere pollution of seismic signals (e.g. Agnew \& Berger 1978), the ambient noise field is now widely recognized as a valuable carrier of information on 3-D Earth structure. In this context, the sources of seismic noise have moved into the focus of scientific attention - as indicators of ocean wave climate, as a limiting factor that controls accuracy and precision of noise tomography and again as a nuisance in noise-based subsurface monitoring.

Since the developments of Sabra et al. (2005) and Shapiro et al. (2005), surface wave tomography based on ambient noise correlations has developed into a standard technique. While the resulting tomographic models are broadly consistent with those from earthquake tomography (e.g. Stehly et al. 2009; Verbeke et al. 2012), the technique relies on the hardly verifiable assumption that noise correlations converge to the Green's function between a pair of receivers. The necessary requirements are not generally justified for the Earth's ambient noise, in particular due to an unfavourable distribution of noise sources in many regions (e.g. Froment et al. 2010). An anisotropic source distribution poses several obstacles to the retrieval of the Green's function: First, traveltimes can be biased beyond the measurement error (Tsai 2009; Yao \& Van Der Hilst 2009; Froment et al. 2010; Delaney 2015). Second, spurious 'precursory' arrivals may occur due to noise incident at high angles to the station-station line or to intermodal cross terms (Kimman \& Trampert 2010; Tian \& Ritzwoller 2015). Third, subjective processing and data manipulation may distort the waveforms, introducing measurement errors and limiting reproducibility (Fichtner 2014). Finally, depending on the nature and location of noise sources, higher surface wave modes may be incorrectly excited, thereby severely limiting the depth resolution of ambient noise surface wave tomography (Halliday \& Curtis 2008; Kimman \& Trampert 2010). The first two problems may be mitigated by the presence of well-distributed scatterers that act as secondary sources. However, a strongly scattering medium is only found in specific regions of study and is dependent on the frequency range analysed. Thus, its presence cannot be assumed in general.

To limit the pollution of tomographic images by source distribution effects, unsuitable receiver- receiver azimuths or frequency bands (e.g. Pedersen \& Krüger 2007; Mordret et al. 2013) must 
be excluded, which reduces the amount of exploitable data. While the traveltime biases can often be ignored in a crustal environment where strong heterogeneities dominate traveltime residuals (Yang \& Ritzwoller 2008; Froment et al. 2010; Kimman \& Trampert 2010; Fichtner 2014, 2015), the amplitudes of noise correlations are of greater concern. Specifically, attenuation studies based on noise require knowledge of the influence of noise sources on crosscorrelation amplitudes (e.g. Hanasoge 2013). Furthermore, the waveform distortions induced by heterogeneous noise source distributions currently prevent reliable full-waveform inversion based on ambient noise correlations (Fichtner 2014; Hanasoge 2014).

To improve ambient noise tomography further, the heterogeneous distribution of noise sources should be taken into account. This requires either physical theories for noise generation or methods to invert seismic data for the distribution of noise sources in time and frequency. Here, we propose a first step towards the latter. Source imaging and inversion and the study of physical processes generating ambient noise benefit from each other. We briefly summarize key findings on physical generation mechanisms in the next paragraph.

\subsection{Physical theories for seismic noise excitation}

While the excitation mechanisms for Rayleigh waves in the microseismic frequency band are well understood, the origin of the Earth's hum and of ambient Love waves are currently less well known. For the ubiquitous and energetic microseismic noise peaks, the primary and secondary generation mechanisms described by LonguetHiggins (1950) and Hasselmann (1963) are well accepted. More recent studies succeeded to model time-dependent noise power spectra (e.g. Ardhuin et al. 2011; Stutzmann et al. 2012) and have shown that local bathymetry controls the potential to excite noise at different frequencies within the microseismic band (Gualtieri et al. 2013; Sergeant et al. 2013).

The excitation mechanism of the Earth's hum has been the subject of considerable debate. Various authors succeeded in locating seasonally varying source areas (Rhie \& Romanowicz 2004, 2006; Nishida \& Fukao 2007), but two main issues remain unresolved. The first one is the importance of the atmosphere as hum source. While Rhie \& Romanowicz (2004) suggest that the cause of the hum is ocean wave interaction with the solid Earth, normal-mode based hum source inversions by Nishida \& Fukao (2007) and Nishida (2014) indicated that acoustic resonance between solid Earth and atmosphere is responsible for the lowermost hum band excitation. The second point is the mechanism by which ocean wave energy is transferred to seismic waves. Traer \& Gerstoft (2014) proposed a model in which hum is excited by a mechanism similar to secondary microseisms. Recently, Ardhuin et al. (2015) have shown that the primary mechanism explains observations from the hum to the primary microseism frequency band. Their opposing theories that hum is excited ubiquitously in shallow waters, or that it is primarily excited close to the shelf breaks, call for a source imaging technique capable of distinguishing these options.

So far, relatively little is known about the origin of Love waves in seismic noise (studies include Fukao et al. 2010; Hadziioannou et al. 2012; Nishida 2014). This holds particularly true for longperiod Love waves, although they contribute significantly to the Earth's hum (Kurrle \& Widmer-Schnidrig 2008). Thus, while much is understood about the excitation of ambient seismic noise, source imaging can still contribute new insights. We next summarize state of the art noise source imaging techniques and key findings.

\subsection{Observations of noise sources}

A well-established method for locating noise sources is beamforming (e.g. Schulte-Pelkum et al. 2004; Bromirski \& Gerstoft 2009; Kurrle \& Widmer-Schnidrig 2006; Rhie \& Romanowicz 2004, 2006; Traer et al. 2012; Reading et al. 2014; Gal et al. 2015). Azimuth and propagation speed which yield the largest beam power at an array are interpreted as directions of arrival of the main noise component. Beamforming is used on all scales: Rhie \& Romanowicz (2004) estimated incidence angles of seismic hum using a fixed propagation velocity, and thereby succeeded to map large-scale hum excitation areas. Using beamforming with variable propagation velocity, Schulte-Pelkum et al. (2004) found evidence for the strong directionality of ambient noise, for the importance of wave propagation effects on noise recording and for the excitation of microseismic noise by distant storms. While beamforming is conceptually simple and fast, the direction-dependent beam power is only qualitatively related to the actual power-spectral density (PSD) of the noise sources. It furthermore relies on a plane-wave assumption that may not be generally valid in the complex Earth.

Schulte-Pelkum et al. (2004) and Chevrot et al. (2007) compared results from beamforming to particle motion polarization analysis. Directions of arrival estimated from polarization analysis showed considerably more scatter, and results were only approximately consistent with beamforming results. However, polarization analysis is able to constrain the direction of arrival with only one three-component station. Chevrot et al. (2007) also attempted to combine direction-of-arrival estimates from array and polarization analysis with attenuation estimates to locate sources precisely. However, numerous parameters needed to be fit to model absolute noise amplitudes, including site response and source energy. In addition, they allowed for one location along the great-circle propagation path between source and array only. Therefore, they identified very sharply determined source regions, but could not quantify the degree of uncertainty of these results.

Studies aimed at wave climate reconstruction from microseismic noise recordings have identified source areas through high correlation between the time series of noise power at seismic stations and significant wave height at buoys (e.g. Bromirski 2001; Essen et al. 2003).

All these observations have been obtained directly from noise time series. However, as Tian \& Ritzwoller (2015) justly mention, ambient noise tomography is done on stacks of noise crosscorrelation functions of the ambient noise. The processing and stacking involved to obtain these functions can change the way the source distribution affects the observations; and there are only few studies to date which estimate the source distribution directly from cross-correlation functions. All previous studies performed to this aim have used cross-correlation asymmetry to deduce directions of arrival (e.g. Stehly et al. 2006; Yang \& Ritzwoller 2008; Tian \& Ritzwoller 2015).

Asymmetric noise cross-correlation functions, where either the causal or the anticausal branch contains a higher-amplitude signal, are observed when sources are dominantly located behind one station only. Stehly et al. (2006) inferred the noise source direction directly from the normalized noise amplitudes of Rayleigh wave arrivals, whereas Yang \& Ritzwoller (2008) and Tian \& Ritzwoller (2015) used the signal-to-noise ratio of both branches. Both Stehly et al. (2006) and Yang \& Ritzwoller (2008) presented a global study of noise-correlation asymmetry in different frequency bands based on several regional arrays. Stehly et al. (2006) found that results for primary and secondary microseisms differed and concluded that 
secondary microseisms mainly originate at nearby coastlines, while primary microseisms originate in the deep ocean. Contrarily, Yang \& Ritzwoller (2008) argued that sources are mainly coastal for both frequency bands and observed differences merely caused by propagation and attenuation effects. Two main difficulties arise in resolving the discrepancy of these studies. The first is that both methods provide directions of arrival, but only very approximate or no constraints on how far away the sources are. The second complication is that different processing strategies were applied (in particular, Stehly et al. (2006) used one-bit normalization), which has been shown to emphasize contributions of different noise sources to the final correlation (e.g. Cupillard \& Capdeville 2010).

In a more recent study, Tian \& Ritzwoller (2015) investigated microseisms observed at ocean-bottom seismometers on the Juan de Fuca plate and at continental stations on the adjacent West Coast of the US. Their data support secondary microseism generation in Pacific deep waters, and primary microseism generation in nearby shallow waters as well as by more distant sources in the Atlantic and Central to South Pacific. The authors noted that their correlation asymmetry method-similar to the one used by Stehly et al. (2006) - is currently the only technique that images noise sources as noise correlations 'see' them.

\subsection{Motivation and outline: ray theory imaging of noise sources using cross-correlation asymmetry}

Here we propose an improved cross-correlation based noise source imaging technique. We require this technique to (i) operate on noise correlation data sets directly and (ii) use an observable that can be measured robustly, modelled numerically and related directly to variations in the PSD distribution of the noise sources.

This paper is organized as follows. In Section 2, we review the modelling of noise cross-correlations and the computation of noise source sensitivity kernels. In Section 2.3, we propose to use the logarithmic energy ratio of the causal and anticausal cross-correlation branches as measurement to infer the noise source distribution. Sensitivity kernels for this measurement with respect to the PSD distribution of noise sources in space and frequency are introduced in the same section. As an intermediate step towards a full 3-D inversion for noise sources, we derive a ray-theoretical simplification of the noise source kernels. This theoretical part forms the foundation of a fast method for noise source imaging that produces physically meaningful results, consistent with previous studies in the microseismic and hum frequency bands. Applications to real data can be found in Section 3, where we illustrate the proposed ray theory imaging of noise sources with examples of globally recorded hum, and microseismic noise in the Western Mediterranean and the Swiss Digital Seismic Network. Finally, in Section 4 we discuss the benefits and limitations of our method, and we place it in the context of the ongoing transition from ray-based to finite-frequency tomography.

\section{THEORY FOR NOISE SOURCE I M A G I N G}

\subsection{Modelling noise correlation functions}

To set the stage for subsequent developments, we provide a condensed review of noise correlation modelling: To forward model interstation correlations of ambient noise, we adopt the method developed by Woodard (1997) in helioseismology and recently applied in terrestrial seismology by various authors (e.g. Tromp et al. 2010; Hanasoge 2013, 2014; Nishida 2014). For the developments in Sections 2.1 and 2.2, interested readers are also referred to Fichtner (2014, 2015).

For notational convenience, we work in the frequency domain, where the $i$ th component of the seismic wavefield $u_{i}(\mathbf{x}, \omega)$, the Green's function $G_{i n}(\mathbf{x}, \mathbf{y}, \omega)$ and the noise sources $N_{n}(\mathbf{y}, \omega)$ are connected by the representation theorem (e.g. Aki \& Richards 2002)

$u_{i}(\mathbf{x}, \omega)=\int_{\oplus} G_{i n}(\mathbf{x}, \mathbf{y}, \omega) N_{n}(\mathbf{y}, \omega) \mathrm{d} \mathbf{y}$.

The integral is over the volume of the Earth $\oplus$. Under the assumption that noise sources are confined to Earth's surface, this volume integral can be reduced to a surface integral, but this is not strictly necessary for the following derivation. The cross-correlation of wavefields $u_{i}, u_{j}$ recorded at positions $\mathbf{x}=\mathbf{x}_{1}$ and $\mathbf{x}=\mathbf{x}_{2}$, respectively, can be expressed as

$$
\begin{aligned}
\mathcal{C}_{i j}\left(\mathbf{x}_{1}, \mathbf{x}_{2}\right)=u_{i}\left(\mathbf{x}_{1}\right) u_{j}^{*}\left(\mathbf{x}_{2}\right) \\
\quad=\int_{\oplus} \int_{\oplus} G_{i n}\left(\mathbf{x}_{1}, \mathbf{y}_{1}\right) N_{n}\left(\mathbf{y}_{1}\right) G_{j m}^{*}\left(\mathbf{x}_{2}, \mathbf{y}_{2}\right) N_{m}^{*}\left(\mathbf{y}_{2}\right) \mathrm{d} \mathbf{y}_{1} \mathrm{~d} \mathbf{y}_{2},
\end{aligned}
$$

where $u_{j}^{*}$ denotes the complex conjugate of $u_{j}$. Dependencies on $\omega$ are omitted in the interest of succinctness whenever possible. Due to the transient nature of noise sources, $\mathcal{C}_{i j}\left(\mathbf{x}_{1}, \mathbf{x}_{2}\right)$ is generally different when data from different time intervals are used. We therefore consider the expectation

$$
\begin{aligned}
\mathbb{E} & {\left[\mathcal{C}_{i j}\left(\mathbf{x}_{1}, \mathbf{x}_{2}\right)\right]=C_{i j}\left(\mathbf{x}_{1}, \mathbf{x}_{2}\right) } \\
& =\int_{\oplus} \int_{\oplus} G_{i n}\left(\mathbf{x}_{1}, \mathbf{y}_{1}\right) G_{j m}^{*}\left(\mathbf{x}_{2}, \mathbf{y}_{2}\right) \mathbb{E}\left[N_{n}\left(\mathbf{y}_{1}\right) N_{m}^{*}\left(\mathbf{y}_{2}\right)\right] \mathrm{d} \mathbf{y}_{1} \mathrm{~d} \mathbf{y}_{2},
\end{aligned}
$$

that can be approximated by the stack over many time intervals, for instance daily windows throughout a year. In eq. (3), the term $\mathbb{E}\left[N_{n}\left(\mathbf{y}_{1}\right) N_{m}^{*}\left(\mathbf{y}_{2}\right)\right]$ denotes the expected correlation of noise sources at positions $\mathbf{y}_{1}$ and $\mathbf{y}_{2}$. We make the approximation

$\mathbb{E}\left[N_{n}\left(\mathbf{y}_{1}\right) N_{m}^{*}\left(\mathbf{y}_{2}\right)\right]=S_{n m}\left(\mathbf{y}_{1}\right) \delta\left(\mathbf{y}_{1}-\mathbf{y}_{2}\right)$,

which simplifies eq. (3) to

$C_{i j}\left(\mathbf{x}_{1}, \mathbf{x}_{2}\right)=\int_{\oplus} G_{i n}\left(\mathbf{x}_{1}, \mathbf{y}\right) G_{j m}^{*}\left(\mathbf{x}_{2}, \mathbf{y}\right) S_{n m}(\mathbf{y}) \mathrm{d} \mathbf{y}$.

The above approximation (eq. 3) means that only sources that are exactly collocated contribute to the stacked correlation. In practice, noise sources may be extended, and consequently the approximation made above may limit the applicability of the method in cases where noise sources are correlated over distances that are large compared to the seismic wavelength.

The Hermitian matrix $S_{n m}$ is the PSD of the noise sources as a function of position and frequency. The PSD is real-valued when the off-diagonal elements of $S_{n m}$ are zero, that is when different noise source components are uncorrelated. Eq. (5) provides a model for the forward calculation of interstation correlation functions for arbitrary distributions of the noise PSD in both space and frequency. The forward model is therefore free of assumptions on wavefield equipartitioning or isotropic source distributions needed to ensure equality of correlations and Green's functions (e.g. Lobkis \& Weaver 2001; Wapenaar 2004; Weaver \& Lobkis 2004; Wapenaar \& Fokkema 2006; Sánchez-Sesma \& Campillo 2006). 


\subsection{Sensitivity kernels for the power-spectral density distribution of noise sources}

The forward model in eq. (5) provides synthetic correlation functions $C_{i j}\left(\mathbf{x}_{1}, \mathbf{x}_{2}\right)$ that can be compared to observed correlation functions $C_{i j}^{0}\left(\mathbf{x}_{1}, \mathbf{x}_{2}\right)$ with the help of a suitably chosen misfit functional $\chi$. An infinitesimal perturbation of the PSD, $\delta S_{n m}$, induces an infinitesimal perturbation of the misfit, $\delta \chi$. In Appendix A1, we show that $\delta \chi$ can generally be written in the form

$\delta \chi=2 \operatorname{Re} \int_{0}^{\infty} \delta C_{i j}\left(\mathbf{x}_{1}, \mathbf{x}_{2}, \omega\right) f(\omega) \mathrm{d} \omega$,

The term $f(\omega)$, which we refer to as the adjoint source, depends on the specific choice of the measurement (such as, for example, traveltime or amplitude difference) and the misfit functional $\chi$ (such as, for example, the $L_{2}$-misfit). In this paragraph, we keep $f(\omega)$ general, and specify the measurement and misfit later on (in Section 2.3).

The misfit variation $\delta \chi$ depends on the perturbation of the correlation $\delta C_{i j}$, with respect to the model parameters, that is, the source PSD. Writing the perturbation as a function of the source PSD perturbation (assuming that the structure does not vary in the time interval we consider), yields:

$\delta C_{i j}\left(\mathbf{x}_{1}, \mathbf{x}_{2}, \omega\right)=\int_{\oplus} G_{i n}\left(\mathbf{x}_{1}, \mathbf{y}, \omega\right) G_{j m}^{*}\left(\mathbf{x}_{2}, \mathbf{y}, \omega\right) \delta S_{n m}(\mathbf{y}, \omega) \mathrm{d} \mathbf{y}$,

Introducing this perturbation of the correlation into eq. (6), gives

$$
\begin{aligned}
\delta \chi= & 2 \operatorname{Re} \int_{0}^{\infty} \int_{\oplus} f(\omega) G_{i n}\left(\mathbf{x}_{1}, \mathbf{y}, \omega\right) \\
& \times G_{j m}^{*}\left(\mathbf{x}_{2}, \mathbf{y}, \omega\right) \delta S_{n m}(\mathbf{y}, \omega) \mathrm{d} \omega \mathrm{d} \mathbf{y} \\
= & \int_{0}^{\infty} \int_{\oplus} K_{n m}(\mathbf{y}, \omega) \delta S_{n m}(\mathbf{y}, \omega) \mathrm{d} \omega \mathrm{d} \mathbf{y} .
\end{aligned}
$$

The sensitivity kernel

$K_{n m}(\mathbf{y}, \omega)=2 \operatorname{Re} f(\omega) G_{i n}\left(\mathbf{x}_{1}, \mathbf{y}, \omega\right) G_{j m}^{*}\left(\mathbf{x}_{2}, \mathbf{y}, \omega\right)$

represents the first-order change of the measured misfit in response to a perturbation of the noise source PSD. We can now briefly exemplify why $f(\omega)$ is referred to as adjoint source. In eq. (9), the term

$f(\omega) G_{i n}\left(\mathbf{x}_{1}, \mathbf{y}, \omega\right)=\int_{\oplus} f(\omega) G_{i n}\left(\mathbf{y}, \mathbf{x}_{1}, \omega\right) \delta\left(\mathbf{x}_{1}\right) \mathrm{d} \mathbf{x}_{1}$

may be interpreted in terms of an adjoint wavefield excited by $f(\omega)$ at receiver position $\mathbf{x}_{1}$ (and can be calculated numerically as such). This establishes an analogy with the adjoint-based computation of sensitivity kernels for Earth structure (e.g. Tarantola 1988; Tromp et al. 2005; Fichtner et al. 2006). If $\delta S_{n m}$ is a unit perturbation to the source PSD, then according to eq. (8) the kernel informs us about the spatial shape of the resulting misfit perturbation.

\subsection{The measurement: causal/anticausal asymmetry}

Eq. (9) is valid for all differentiable misfit functionals $\chi$. Now, we chose a specific measurement and a specific misfit, $\chi$. This determines a specific adjoint source $f(\omega)$, and therefore the shape and amplitude of the sensitivity kernel $K_{n m}$. We first explain why we chose the measurement $A$, which is the causal/anticausal asymmetry. We then derive the sensitivity kernel corresponding to this measurement, and to an $L_{2}$-misfit. In the following sections, the kernel will be strongly simplified for application examples.

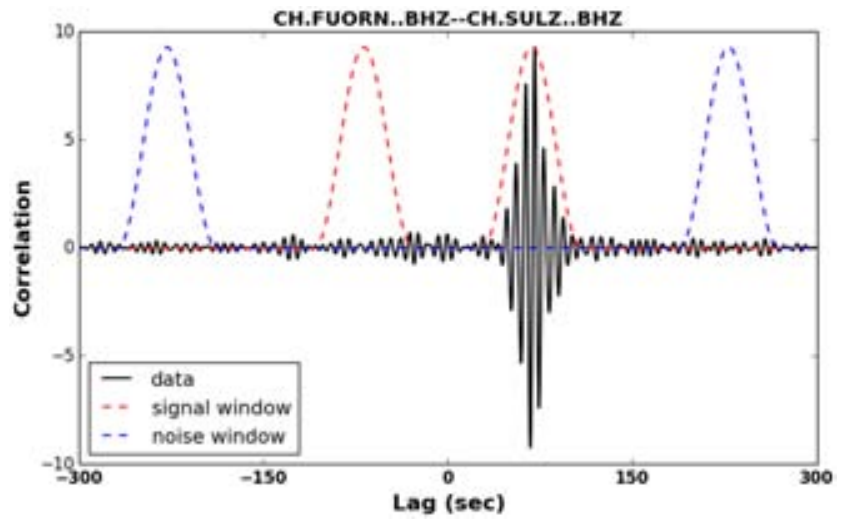

Figure 1. Selection of the signal and noise windows on a correlation between the two Swiss stations FUORN and SULZ during January 2014. Measurements of correlation asymmetry are made for the signal windows in red, which are Hann windows centred on the estimated arrivals of the causal and anticausal Rayleigh waves. The signal-to-noise ratio is estimated for two noise windows in blue. These are separated by one window length from the signal window. More details on the estimation of the signal-to-noise ratio and its role in the measurement process are provided in Section 3.1 where we consider specific examples. Note that the correlation stack amplitude is arbitrary because windows are normalized prior to stacking.

To image the geographic distribution of noise sources $S_{n m}$, we require $\chi$ to be insensitive, at least to first order, to the presence of unmodelled 3-D Earth structure. While the amplitudes of correlation waveforms are primarily sensitive to the geographic distribution of noise sources (e.g. Hanasoge 2013, 2014), they are also affected by focusing and defocusing in the presence of 3-D structure, as well as by viscoelastic attenuation (e.g. Prieto et al. 2011). To circumvent the difficulty of interpreting amplitudes themselves, we quantify noise correlation asymmetry in terms of the logarithmic energy ratio of the causal and anticausal parts.

For this we first consider a time window $w_{+}(t)$, centred around a synthetically computed correlation waveform on the positive lag axis. Its mirrored counterpart on the negative lag axis is denoted by $w_{-}(t)$. An exemplary window selection is shown in Fig. 1 . The correlation asymmetry is then

$A=\ln \frac{\int_{-\infty}^{\infty}\left[w_{+}(t) C_{i j}(t)\right]^{2} \mathrm{~d} t}{\int_{-\infty}^{\infty}\left[w_{-}(t) C_{i j}(t)\right]^{2} \mathrm{~d} t}$.

The asymmetry of observed correlation functions $A^{0}$ is defined analogously to eq. (11), which allows us to quantify the discrepancy between observations and synthetics in terms of the total $L_{2}$ misfit

$\chi_{\mathrm{tot}}=\sum_{n=1}^{N}\left[\frac{1}{2}\left[A_{n}-A_{n}^{0}\right]^{2}\right]$

where the sum is over all pairs of receivers, and $A_{n}$ and $A_{n}^{0}$ are the modelled and observed logarithmic asymmetry at the $n$th receiver pair. This is the summed misfit for all measurements; the sum is now omitted, and one measurement only is considered, for notational convenience:

$\chi=\frac{1}{2}\left[A-A^{0}\right]^{2}$.

A homogeneous reference noise source distribution for the forward problem solution (3) is assumed to produce perfectly symmetric synthetic correlation functions with $A=0$. Higher energy on the causal side corresponds to positive asymmetry ( $A$ or $A^{0}>0$ ), and vice versa. 
As shown in Appendix A2, the measurement (11) and misfit definition (13) generate the following adjoint source $f(\omega)$, needed for the computation of noise source sensitivity kernels according to eq. (9):

$$
\begin{aligned}
f(\omega)=\left(A-A^{0}\right)\left[\frac{1}{\pi E_{+}}\right. & \left(w_{+}^{2}(\omega) * C_{i j}(\omega)\right)^{*} \\
& \left.\quad-\frac{1}{\pi E_{-}}\left(w_{-}^{2}(\omega) * C_{i j}(\omega)\right)^{*}\right],
\end{aligned}
$$

where $E_{+}$and $E_{-}$denote the energies in the causal and anticausal windows, respectively:

$$
\begin{aligned}
& E_{+}=\int_{-\infty}^{\infty}\left[w_{+}(t) C_{i j}(t)\right]^{2} \mathrm{~d} t, \\
& E_{-}=\int_{-\infty}^{\infty}\left[w_{-}(t) C_{i j}(t)\right]^{2} \mathrm{~d} t .
\end{aligned}
$$

The symbol $*$ denotes convolution, and $w_{+}^{2}(\omega)$ is the Fourier transform of the squared time window $w_{+}^{2}(t)$. Combining eqs (9) and (15), gives an explicit expression for the noise source kernel $K_{n m}$ :

$$
\begin{aligned}
K_{n m}(\mathbf{y}, \omega)= & \left(A-A^{0}\right)\left[\frac{1}{\pi E_{+}}\left(w_{+}^{2}(\omega) * C_{i j}(\omega)\right)^{*}\right. \\
& \left.-\frac{1}{\pi E_{-}}\left(w_{-}^{2}(\omega) * C_{i j}(\omega)\right)^{*}\right] G_{i n}\left(\mathbf{x}_{1}, \mathbf{y}, \omega\right) \\
& \times G_{j m}^{*}\left(\mathbf{x}_{2}, \mathbf{y}, \omega\right) .
\end{aligned}
$$

While the term $G_{i n}\left(\mathbf{x}_{1}, \mathbf{y}, \omega\right) G_{j m}^{*}\left(\mathbf{x}_{2}, \mathbf{y}, \omega\right)$ determines the regions where sensitivity can be non-zero, the term in square brackets controls the sign and amplitude of sensitivity within these regions as a function of frequency.

\subsection{Simplified kernels}

Eq. (16) for the noise source kernel $K_{n m}$, based on the logarithmic energy ratio $A$, is valid in 3-D viscoelastic media where both singleand mixed-component correlations are considered. To develop a computationally inexpensive tool for rapid estimation of the noise source distributions prior to a detailed inversion, we adopt two simplifications: (i) We limit the starting model to a homogeneous noise source distribution within a 2-D structurally homogeneous medium, and (ii) we reduce spatially extended sensitivity kernels to rays. These simplifications are detailed below.

\subsubsection{2-D homogeneous media}

In the first stage of simplifications, we choose a homogeneous noise source distribution as reference, and a homogeneous medium of wave propagation. Given this homogeneous source distribution and homogeneous medium, it follows that $E_{+}=E_{-}=E$ and $A=0$. We limit the analysis to vertical-component correlations. Furthermore, we use 2-D wave propagation as an analogue for single-mode surface wave propagation. A similar approach was taken by Hanasoge $(2013,2014)$ to study noise correlation amplitude kernels. With the noise source distribution $S(\omega)$ being constant in space, eqs (5) and (16) are simplified to

$$
C\left(\mathbf{x}_{1}, \mathbf{x}_{2}\right)=S \int G\left(\mathbf{x}_{1}, \mathbf{x}\right) G^{*}\left(\mathbf{x}_{2}, \mathbf{x}\right) \mathrm{d} \mathbf{y}
$$

and

$$
\begin{aligned}
K(\mathbf{x}, \omega)= & -\frac{A^{0}}{\pi E}\left[\left(w_{+}^{2}(\omega) * C(\omega)\right)^{*}-\left(w_{-}^{2}(\omega) * C(\omega)\right)^{*}\right] \\
& \times G\left(\mathbf{x}_{1}, \mathbf{x}, \omega\right) G^{*}\left(\mathbf{x}_{2}, \mathbf{x}, \omega\right) .
\end{aligned}
$$

In an unbounded 2-D medium with mass density $\rho$ and shear modulus $\mu$, the far-field Green's function is given by

$G(\mathbf{x}, \mathbf{y}, \omega)=-i \frac{1}{4 \rho v^{2}} \sqrt{\frac{2 v}{\pi \omega r}} \mathrm{e}^{-i \frac{\omega}{v} r} \mathrm{e}^{-\frac{\omega r}{2 v Q}} \mathrm{e}^{i \frac{\pi}{4}}$,

In eq. (19), $v=\sqrt{\mu / \rho}$ denotes the phase velocity, $r=|\mathbf{x}-\mathbf{y}|$ denotes the source-receiver distance and $Q$ denotes the quality factor or inverse attenuation. The far-field approximation has no effect on the synthetic correlation functions and the sensitivity kernels at distances of more than a wavelength from each of the receivers, and it greatly simplifies the subsequent analysis. Substituting the Green's function (19) into expression (9) for the sensitivity kernel $K(\mathbf{x})$ gives

$K(\mathbf{x})=\operatorname{Re} \frac{1}{4 \pi \omega \rho^{2} v^{3}} \frac{f}{\sqrt{r_{1} r_{2}}} \mathrm{e}^{i \frac{\omega}{v}\left(r_{2}-r_{1}\right)} \mathrm{e}^{-\frac{\omega}{2 v Q}\left(r_{1}+r_{2}\right)}$,

where we defined $r_{1}=\left|\mathbf{x}-\mathbf{x}_{1}\right|$ and $r_{2}=\left|\mathbf{x}-\mathbf{x}_{2}\right|$. A gallery of noise source kernels for different values of $Q$ and different periods, is shown in Fig. 2. All kernels have a characteristic hyperbolic shape with almost no sensitivity in between the pair of receivers. With increasing frequency, Fresnel zones become narrower, and kernel amplitudes decay more rapidly — as predicted by eq. (20). For instance, at $20 \mathrm{~s}$ period and $Q=100$, the kernel extends approximately $2500 \mathrm{~km}$ on either side of the receiver pair. This is in contrast to the long-period kernel at $100 \mathrm{~s}$ that extends further than $20000 \mathrm{~km}$ for $Q=300$. More examples of noise source kernels for different types of measurements may be found in Tromp et al. (2010) and Hanasoge $(2013,2014)$; more detail on source-structure trade-offs and attenuation-dependent kernels can be found in Fichtner (2015).

\subsubsection{Reduction to ray theory and the imaging concept}

To simplify the noise source kernels geometrically, we collapse sensitivity into an infinitesimally thin ray with amplitude equal to the integral perpendicular to the great-circle connecting the two receivers. In the specific context of the previous 2-D examples where both receivers were located along the $x$-axis, we thus replace $K(\mathbf{x})=K(x, y)$ by

$K_{\text {ray }}(x)=\int K(x, y) \mathrm{d} y$.

In Appendix B, we demonstrate that the amplitude of the ray theory kernel $K_{\text {ray }}$ is given by

$K_{\text {ray }}(x) \approx$ const. $\cdot \mathrm{e}^{-\frac{\omega x}{v Q}}$.

In media without attenuation, that is infinite $Q$, the kernel amplitude along the ray is constant. It follows that the inversion for ambient noise sources in the hypothetical absence of attenuation is an inherently global problem, even when a small local array of receivers is used. The presence of attenuation effectively localizes the kernels closer to the receiver pair. When attenuation is large, perturbations of noise sources at great distance will have little effect on the correlation asymmetry.

A particularly noteworthy aspect of eq. (22) is the fact that the kernel amplitude decays as $\mathrm{e}^{-1 / Q}$ whereas the amplitude of the wavefield decays less rapidly as $\mathrm{e}^{-1 / 2 Q}$. The kernels decay comparatively 

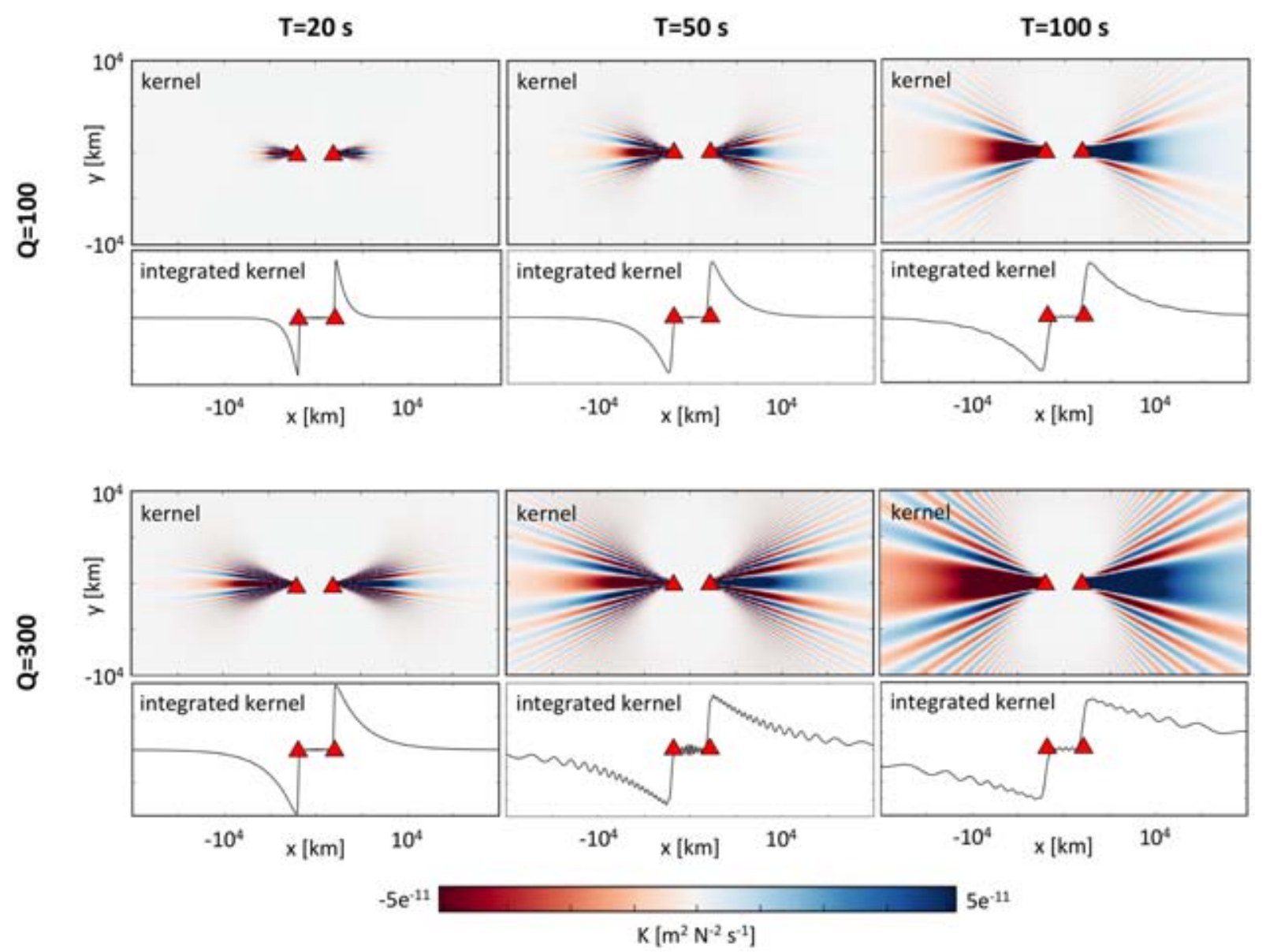

Figure 2. Gallery of noise source kernels for $Q=100$ (top row) and $Q=300$ (bottom row) at periods of 20, 50 and $100 \mathrm{~s}$ (from left to right). Red triangles mark the receiver positions. Plotted below each kernel is its integral in the $y$-direction, which more clearly shows the decay of the kernel amplitude with increasing distance from the receiver pair. With increasing frequencies, Fresnel zones become narrower and decay more quickly. For $Q=100$ and $T=20 \mathrm{~s}$, the kernel extends approximately $2500 \mathrm{~km}$ behind both receivers (top left panel). Sensitivity is practically zero in between the receivers.

rapidly because a product of two wavefields and not a wavefield itself enters the computation of the correlation function.

Based on the ray-theoretical kernels, we can introduce a source imaging concept. This is illustrated in Fig. 3 using the example of a noise correlation between the North American stations GLA and WCI. The noise correlation is strongly asymmetric with larger surface-wave amplitudes on the anticausal branch, thus indicating that energy travels predominantly from west to east. The corresponding noise source kernel multiplied by -1 designates a descent direction, that is the direction of a first update that would place stronger sources to the west of the receiver pair, and weaker sources to the east - assuming a homogeneous reference. The ray-theoretical kernel encodes the same information but in a simplified fashion that enables the rapid estimation of noise source distributions for a large number of receiver pairs, without the need to compute extended finite-frequency kernels for all of them.

For the imaging of noise sources, we scale the ray theoretical kernels such that the kernel value 1 corresponds to the maximum observed asymmetry for the complete noise correlation data set. The ensemble of kernels for all receiver pairs then provides an image of the normalized PSD variation

$$
\delta S_{\mathrm{norm}}=\frac{\Delta S(\mathbf{x})}{\max (\Delta S(\mathbf{x}))}
$$

This image is a scaled version of the first update in a gradient-based optimization of the source PSD. Put in more colloquial terms, the image shows in what direction an optimization of source PSD to fit the amplitude-ratio of the data would look like. It is the first step towards running an inversion for source PSD: the imagesimilar to a colour-coded traveltime misfit plot in a tomographic inversion - enables us to decide whether running an inversion is worthwhile, and it provides a valuable first estimate of the results. Determining the normalization constant would require additional forward modelling steps to determine the optimal step length in the first iteration of a gradient-based optimization; which would be the logic next step beyond the imaging proposed here. In Section 3, we illustrate this imaging concept using a variety of regional and global examples.

\section{APPLICATIONS: IMAGING NOISE SOURCES FROM REGIONAL TO GLOBAL SCALES}

To illustrate the proposed ray theory imaging of noise sources on the basis of asymmetry measurements, we present three real-data examples on different scales. These include (i) noise correlations for globally distributed stations in the hum frequency band, (ii) noise correlations in the microseismic band for stations deployed in the Western Mediterranean and (iii) correlations of microseismic noise 

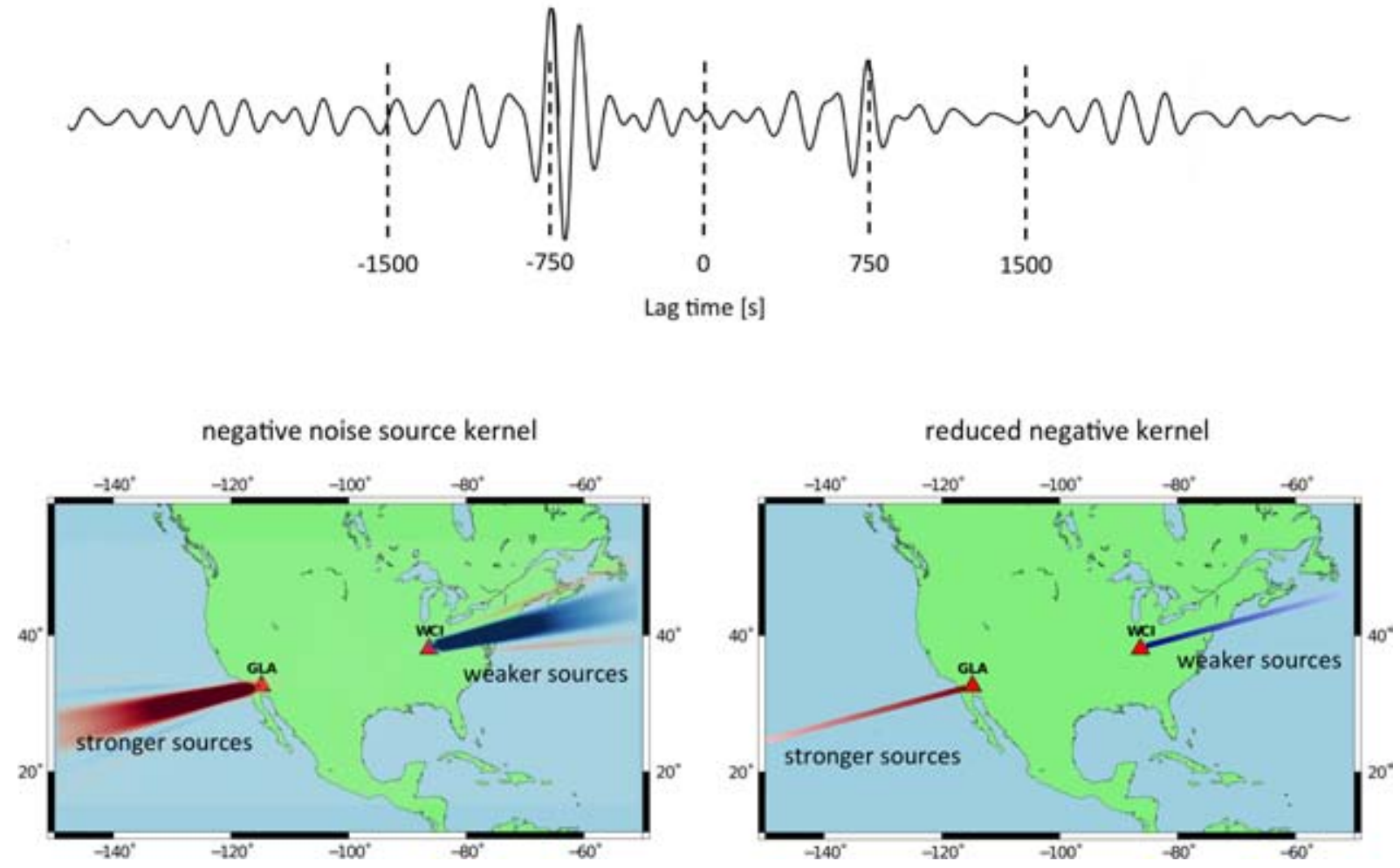

Figure 3. Schematic illustration of the noise source imaging concept. The noise correlation between stations GLA and WCI is strongly asymmetric, indicating that seismic energy propagates predominantly from west to east. The negative noise source kernel, shown in the lower left, constitutes a descent direction, that is, an update to the homogeneous reference that would increase the noise source power-spectral density to the west of the receiver pair and decrease power-spectral density to the east. Shown in the lower right is the ray theoretical kernel that encodes the same information, though in a simplified way that avoids the computation of finite-frequency kernels for a potentially large data set of noise correlations.

recorded at broad-band stations within Switzerland. The Western Mediterranean data originate from permanent stations in Spain, Portugal and France, as well as from temporary deployments in the Iberian Peninsula (IberArray), northern Africa (PICASSO, Universities of Münster and Bristol) and Southern France (PYROPE). More details on the data can be found in Díaz et al. (2009), Chevrot et al. (2014) and Thurner et al. (2014). Station maps for all three examples are shown in Fig. 4. The processing and measurement details for all data sets are described in Section 3.1. This will be followed in Sections 3.2-3.4 by the presentation and discussion of the individual noise source images.

\subsection{Processing, measurements and visualization}

Data provenance and processing are summarized in Table 1. During the processing, we tried to avoid any non-linear operations. We use the classical cross-correlation with geometric normalization. All correlation windows are then stacked linearly. Where necessary, we removed windows containing earthquakes using an sta/lta network coincidence trigger (this is discussed in more detail for each application example).

Given what strong effects the pre-processing scheme applied to ambient noise correlations can have on the results, and how much effort has been spent to investigate different processing strategies (e.g. Bensen et al. 2007; Prieto et al. 2011; Schimmel et al. 2011; Groos et al. 2012; Shen et al. 2012; Boué et al. 2014), this approach may appear unconventional at first sight. However, we do not aim to obtain an empirical Green's function from the noise correlations, but rather a correlation per se that can be forward modelled. The less pre-processing is applied, the more closely the forward model corresponds to the observations.

Moreover, pre-processing usually seeks to isotropize the ambient noise field. This is not conducive to our goal of mapping ambient noise sources. Finally, processing can influence whether local sources or distant sources dominate the correlation (Stehly et al. 2006; Cupillard \& Capdeville 2010). We take a simple approach of correlating and stacking. We potentially sacrifice good signal-to-noise ratios, and weak coherent signals from distant sources, at the benefit of being able to derive the above sensitivity kernels, and to construct forward models of ambient noise correlations.

Processing such as instrument correction, and correlation was undertaken with a parallel processing tool which is based on Python and the obspy seismological toolkit (Beyreuther et al. 2010). Our parallel processing/correlation toolbox is available at https://github.com/echolite/ANTS.

To investigate sources in different frequency bands, we bandpass filter correlations, as summarized in Table 1. With the processing used here, the differences between correlations filtered before or after correlation are negligible. In the interest of greater flexibility, we therefore apply the bandpass filter after correlation and stacking.

To measure correlation asymmetry, we empirically determine Rayleigh wave group traveltimes on record sections of the noise correlations. We then measure energy ratios for Hann windows 

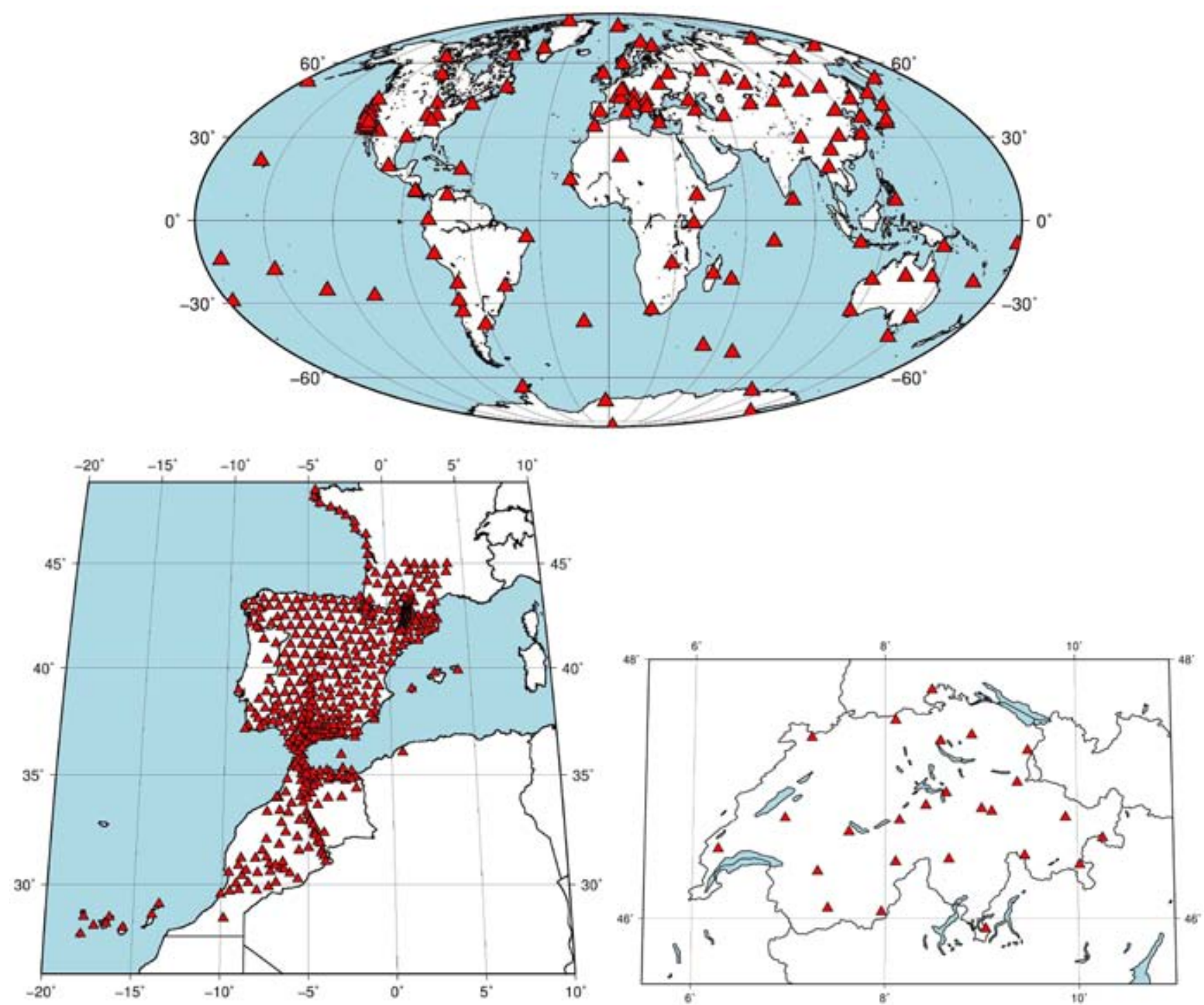

Figure 4. Distribution of seismic stations used in the noise source imaging examples. Top: global distribution of_STS-1 stations used for correlations in the hum frequency band. Bottom: seismic stations in the Western Mediterranean (left), and from the Swiss broad-band network (right).

Table 1. Summary of data provenance and processing for the three real-data examples presented in the following paragraphs.

\begin{tabular}{llll}
\hline Data set & Globally recorded hum & Iber Array & Swiss Nat. Network \\
\hline Time covered & 2004 to 2013 & 2008 to 2011 & January and July 2014 \\
Average stack length & 290 d (Fig. 5), 520 d (Fig. 6) & $434 \mathrm{~d}$ & 24 d \\
Network & IRIS virtual network_STS-1 & IberArray, PICASSO, PYROPE & CH (SDSNet) \\
Processing steps & zero-pad gaps $\leq 60 \mathrm{~s}$ & Fill gaps of $<\approx 5 \mathrm{~min}$ & zero-pad gaps $\leq 2 \mathrm{~s}$ \\
& Decimate to $1 \mathrm{~Hz}$ & Decimate to $5 \mathrm{~Hz}$ & Decimate to $5 \mathrm{~Hz}$ \\
& Correct to ground velocity & Correct to ground velocity & Correct to ground velocity \\
& Windows $32768 \mathrm{~s}$ & Windows $14400 \mathrm{~s}$ & Windows 4096 s \\
& Decimate to $0.1 \mathrm{~Hz}$ & Spectral whitening & Removal of earthquakes \\
& Bandpass filter $0.002-0.05 \mathrm{~Hz}$ & & \\
& Correlation -12000 to $12000 \mathrm{~s}$ & Correlation -3000 to $3000 \mathrm{~s}$ & Correlation -300 to $300 \mathrm{~s}$ \\
Analysed frequency bands & $0.004-0.02 \mathrm{~Hz}$ & $0.05-0.1 \mathrm{~Hz}$ and $0.1-0.2 \mathrm{~Hz}$ & $0.05-0.1 \mathrm{~Hz}$ and $0.1-0.2 \mathrm{~Hz}$ \\
\hline
\end{tabular}

centred on the empirical arrival times on the causal and anticausal branches. To ensure that the main surface wave signal is indeed captured, we visually inspect a subset of the correlations.
We assess measurement quality by comparing the energy in the measurement windows to the energy in two noise windows centred at times where no major arrival is expected. An example is shown in Fig. 1. Measurements where the signal-to-noise ratio, defined here 

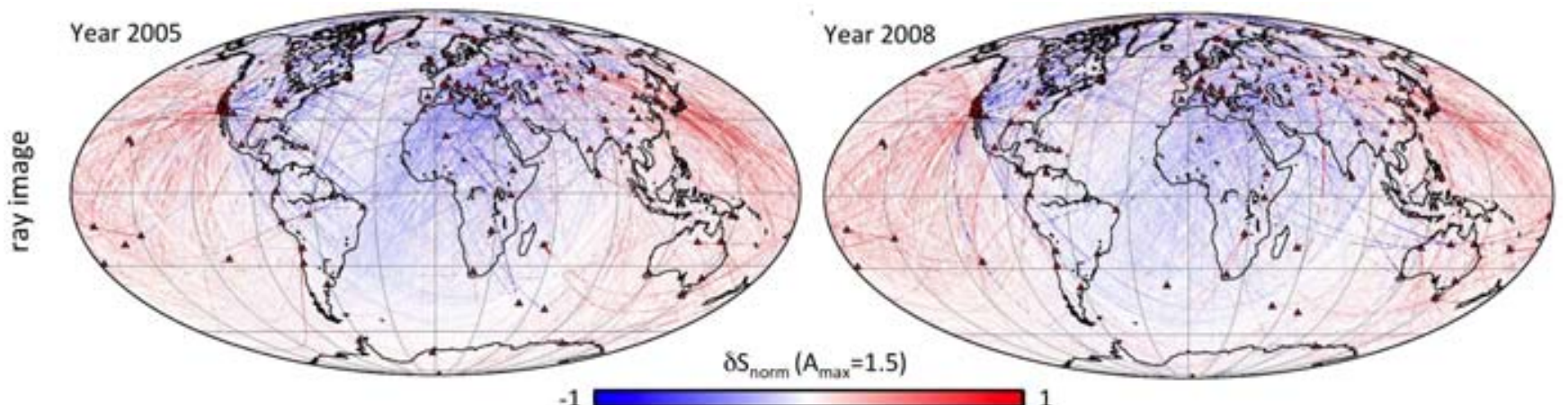

$-1$

1

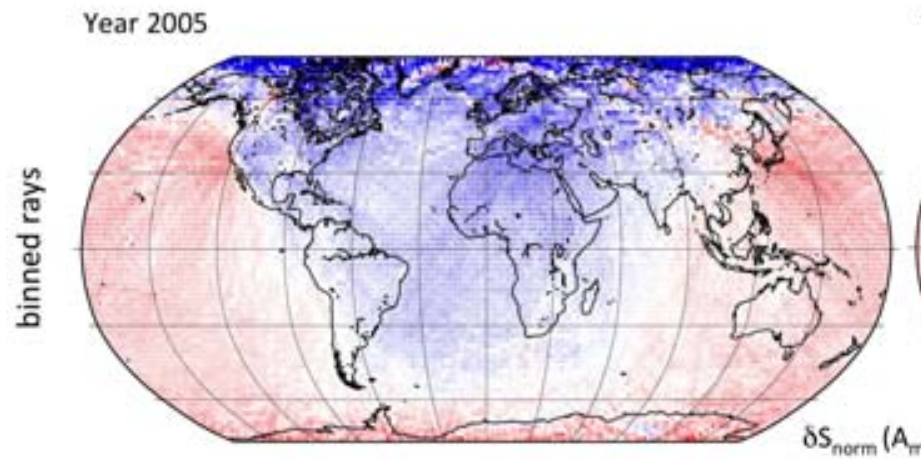

Year 2008

$-1$

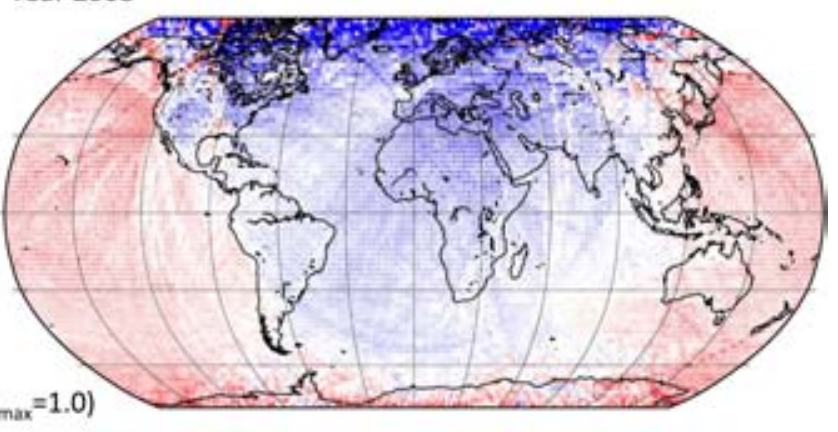

1

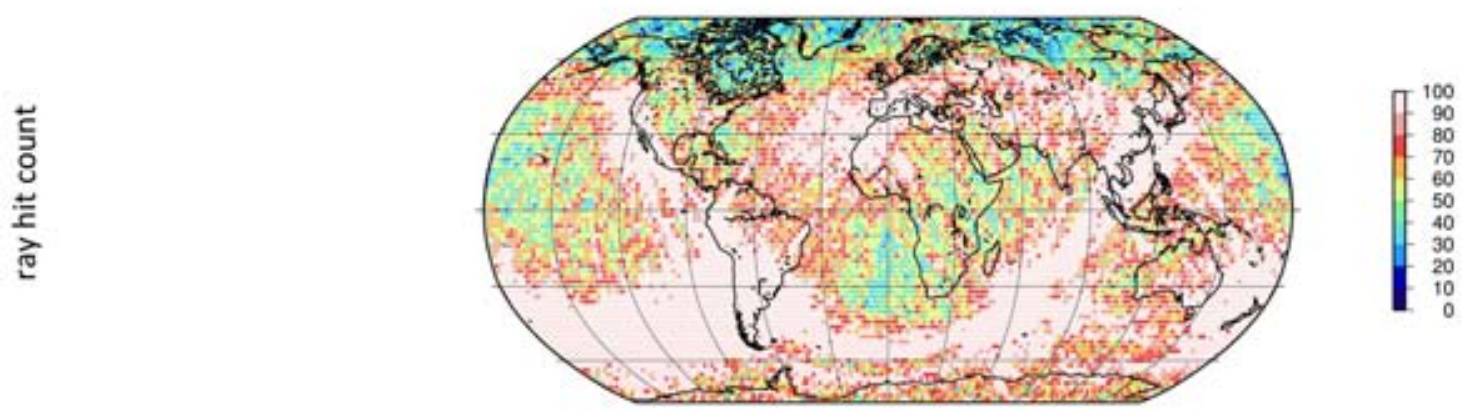

Figure 5. Source images for globally recorded hum in the frequency band from 4 to $20 \mathrm{mHz}$. Top: maximum-normalized power-spectral density of noise sources for year 2005 (left) and year 2008 (right), as defined in eq. (22). The maximum observed asymmetry, $A_{\max }$ is indicated above the colour bar. Ray paths trace the great-circle major-arc between each receiver pair. Middle: as above, but with rays binned and normalized into $2^{\circ} \times 2^{\circ}$ cells. Bottom: number of rays crossing the $2^{\circ} \times 2^{\circ}$ cells.

as the energy ratio of the measurement and noise windows, drops below a pre-defined threshold are excluded. We choose a threshold of 10 for the microseismic regional data and a threshold of 7.5 for long-period global data.

Adopting the imaging concept introduced in Section 2.4.2, we plot all correlation asymmetry measurements on the great-circle major arc connecting the relevant pairs of receivers. This approach is similar to the visualization of traveltime anomalies along ray paths in order to assess their potential in a subsequent traveltime tomography (e.g. Ritsema et al. 2007). To avoid a visual bias in regions where many rays cross, we bin rays into $2^{\circ} \times 2^{\circ}$ bins for the long-period global correlations and into $0.5^{\circ} \times 0.5^{\circ}$ bins for the regional correlations. This is illustrated in Fig. 5.

In the following sections, we show maps of noise source distributions for the global hum, and the microseismic noise in the Western Mediterranean and in the Swiss broad-band network. It is important to keep in mind that these maps show the deviation from a homogeneous reference distribution of sources rather than an absolute PSD distribution, for which the normalization term of eq. (23) would have to be determined.

\subsection{Case I: the sources of globally recorded hum}

Images of seismic hum sources are shown in Figs 5 and 6. Fig. 5 shows hum sources in a broader frequency band $(4-20 \mathrm{mHz})$ for two different years (2005 and 2008). Fig. 6 shows hum sources in a narrow frequency band of 6-8 $\mathrm{mHz}$ for four different months. Each monthly plot contains data from ten times the same month during the years 2004 to 2013, to improve signal-to-noise ratios and detect 'typical' seasonal patterns.

Earthquake signals were not cut out during processing in this case; the resulting maps therefore strictly speaking show effective sources of the correlation wave field including anything recorded. We propose that the large-scale features of these maps can still be interpreted in terms of ambient seismic sources, because (i) a large 

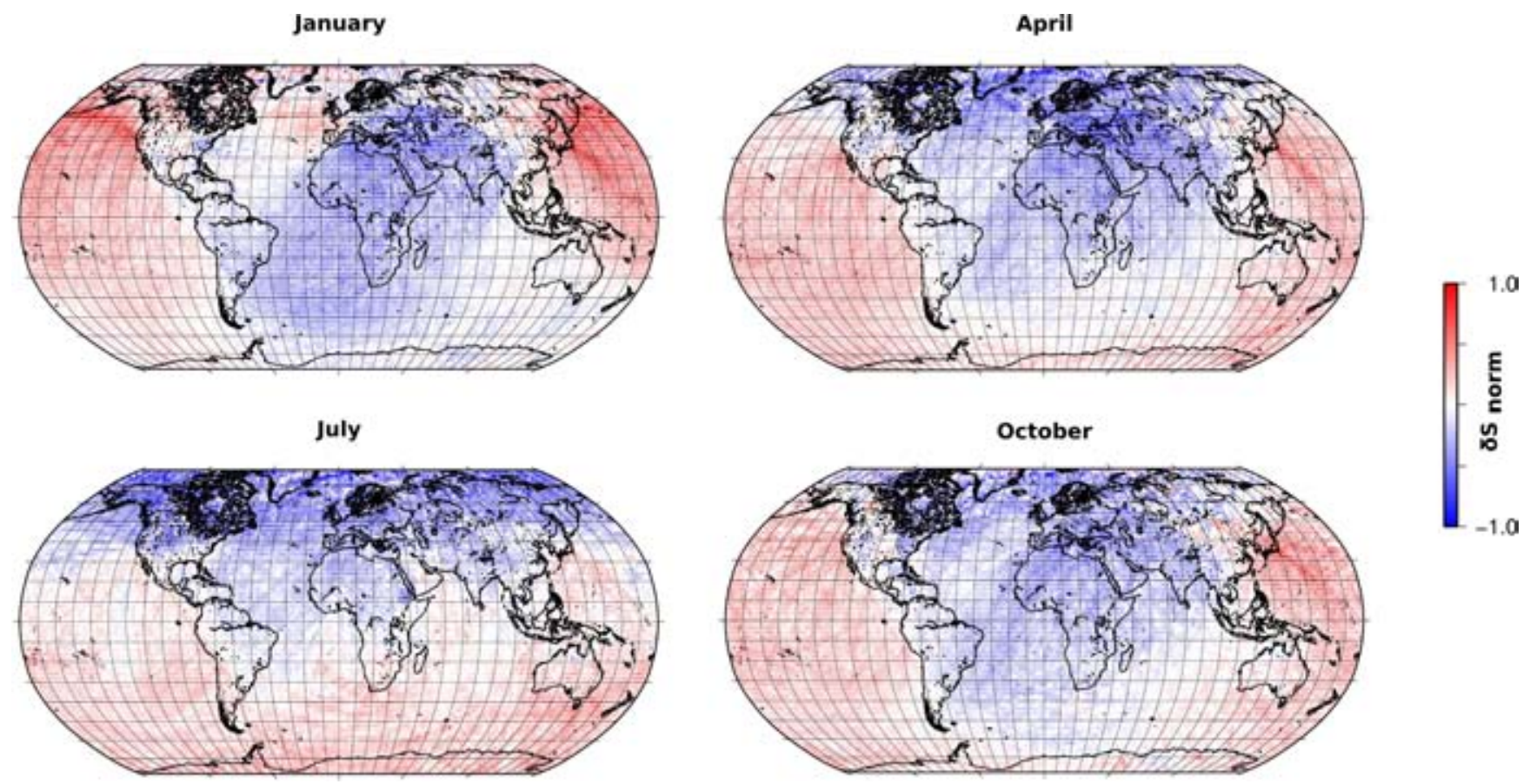

Figure 6. Source images for globally recorded hum in the frequency band 6-8 mHz and for the months January (including all Januarys from 2004 to 2013 ), April, July and October. A distinct seasonal change between January and July is observed where in January, source power is elevated in the Northern Hemisphere and in July, source power is elevated in the Southern Hemisphere, corresponding to the respective winter months.

number of time windows was stacked for each correlation, reducing the influence of any comparatively short signals; (ii) station distances are generally large and preclude highly coherent earthquake signals as would be expected at a single array; an exception are the Berkeley Digital Seismic Network and Southern California Earthquake Center arrays, but excluding these does not change overall map patterns (see Supporting Information Fig. S1); (iii) observed source patterns in Fig. 6 are clearly seasonal and (iv) results obtained with the phase cross-correlation (Schimmel 1999), which emphasizes weak coherent signals, are consistent with the results presented here except for minor details (see Supporting Information Fig. S2).

The images for the years 2005 and 2008 are similar on length scales of $\sim 1000 \mathrm{~km}$ in both the patterns and their amplitudes. This indicates that the sources of hum averaged over one year are stable over time, without being dominated by non-repeatable events. We observe a clear dominance of the Pacific hemisphere, with the strongest sources located in the North Pacific. The sources of hum mapped here are also mostly weaker on the continents than in the oceans. Notable exceptions are continental East Asia and Australia where hum sources are stronger than average, as well as the Atlantic Ocean where sources are relatively weak. Coverage in these regions is good, as shown in the hit count map in the bottom row of Fig. 5. The polar regions should be excluded from interpretations, because the geographic bins are too small to provide stable results.

The hit count map in Fig. 5 as well as the ray image reveal a strong contribution of the Berkeley Digital Seismic Network and the Southern California Seismic Network that contributed a comparatively large number of STS-1 recordings for the study period. However, excluding both networks does not significantly change the hum source images (see Supporting Information Fig. S1).

In Fig. 6, the images for January and July show a strong difference. In January, the strongest increase in source power is located in the North Pacific. A weaker region of increased source power is observed in the North Atlantic. In July, increased source power is mapped in the South Pacific, Indian Ocean and Southern Ocean. April and October show intermediate patterns, which are similar to each other. Our observations are in accord with previous studies on the origin of the Earth's hum. Yang \& Ritzwoller (2008) found that the direction of arrival of the strongest European noise sources at periods $>50 \mathrm{~s}$ invariably points towards sources in the Pacific, as on our yearly images. The North Atlantic, on our source images, also shows elevated source power in January, but comparatively weaker than the North Pacific.

In their global study, Rhie \& Romanowicz (2004) found that hum sources are primarily located in the Northern Pacific during northern hemisphere winter, and in the Southern Ocean during northern hemisphere summer. Similarly, the inversion of cross-spectra by Nishida \& Fukao (2007) revealed that from November to April a spherical harmonic degree-1 pattern with a maximum in the Northern Pacific dominates the hum source distribution. Furthermore, they found that during the northern summer months hum sources are primarily located along the Pacific rims as well as in the Indian Ocean.

Our data for the Earth's hum suggest that the North Atlantic is, during most seasons, a region of weaker than global average noise excitation. This result may be surprising considering the work of Essen et al. (2003) and Kedar et al. (2008) which suggests strong microseismic noise sources in the North Atlantic. However, the excitation mechanism of the Earth's hum and its bathymetric requirements are different from the one of secondary microseisms (Ardhuin et al. 2015). Moreover, predominance of Pacific hum sources is consistent with earlier studies (Rhie \& Romanowicz 2004; Nishida \& Fukao 2007; Yang \& Ritzwoller 2008). Our results indicate that while North Atlantic hum sources do contribute to the excitation of the Earth's hum in northern hemisphere winter, their contribution is less energetic than that from the Pacific. 
single-frequency, $0.05-0.10 \mathrm{~Hz}$

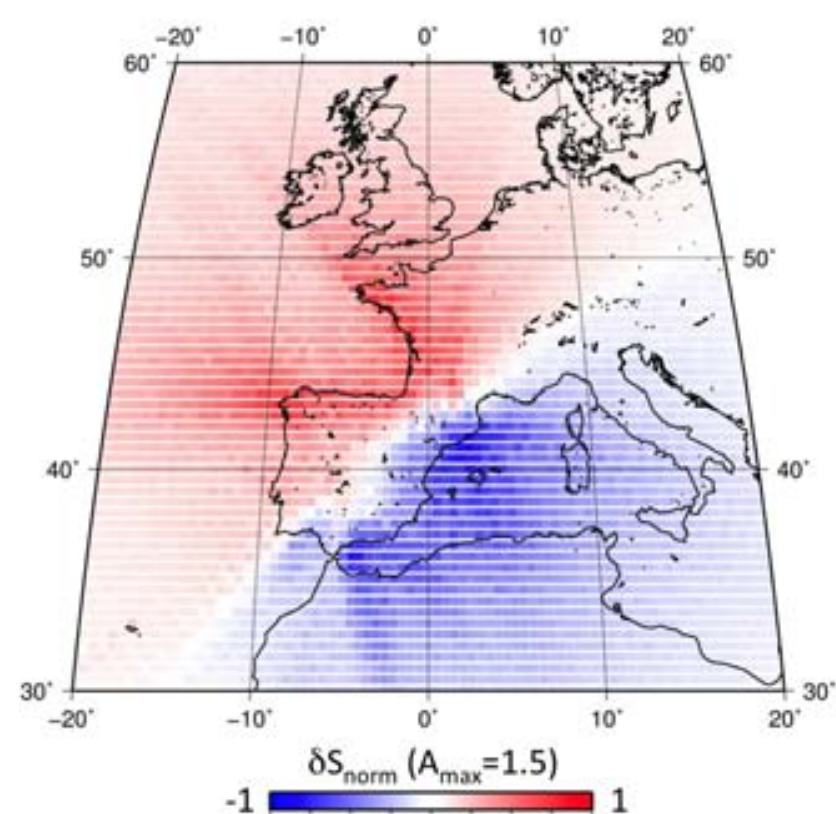

double-frequency, $0.10-0.20 \mathrm{~Hz}$

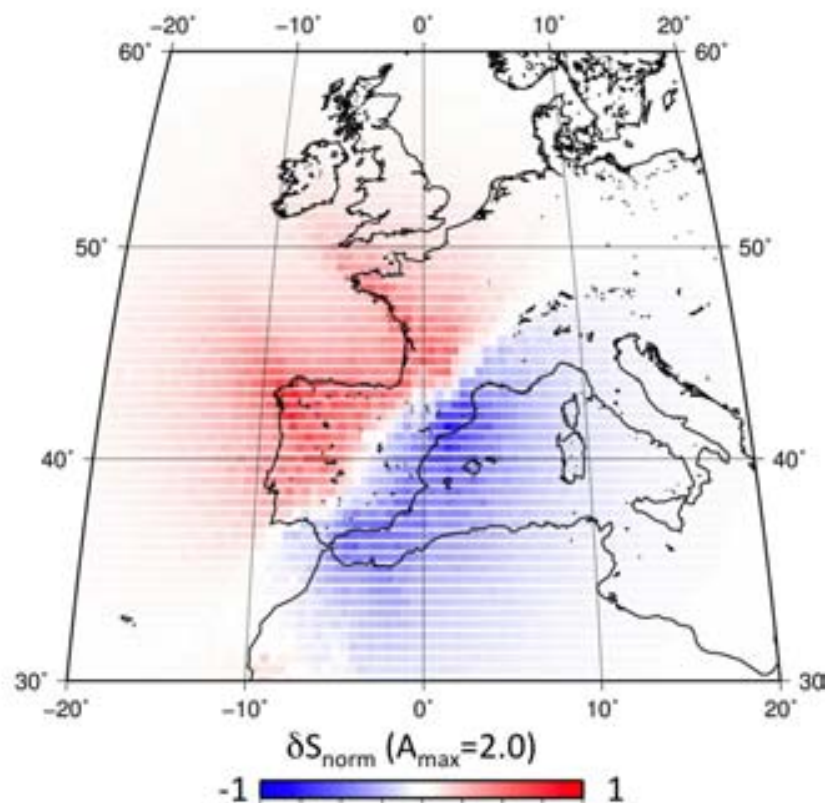

Figure 7. Maximum-normalized power-spectral density distribution of noise sources for ambient noise correlations between Western Mediterranean stations (Díaz et al. 2009) in the single-frequency microseismic band $(0.05-0.10 \mathrm{~Hz}$, left) and the double-frequency microseismic band (0.10-0.20 Hz, right). The maximum observed asymmetries are given in brackets above the colour bars.

\subsection{Case II: microseismic noise in the Western Mediterranean}

Noise source images for the Western Mediterranean correlations are displayed in Fig. 7 for the single-frequency microseismic band $(0.05-0.10 \mathrm{~Hz})$ and the double-frequency microseismic band $(0.10$ $0.20 \mathrm{~Hz}$ ). We observe a distinctly two-sided pattern in both frequency bands, pointing to weaker than average sources in the Western Mediterranean, and stronger than average sources in the Atlantic and along the coast of the Bay of Biscay in particular. In the double-frequency band the maximum observed asymmetry of \pm 4.6 is $\sim 30$ per cent larger than for the single frequency band. This translates to maximum causal/anticausal energy ratios of $\sim 33$ and $\sim 100$, respectively.

The slight differences in the pattern between the single- and double-frequency images mostly result from the faster decay of sensitivity at higher frequencies, already discussed in Section 2.4.2. Attenuation leads to a stronger localization of potential noise sources in the vicinity of the receiver pair. Small differences not related to frequency-dependent sensitivity are limited to the region northeast of the Canary Islands. In the single-frequency band, the Canary Island stations require sources towards south, whereas the doublefrequency microseisms require a weak patch of stronger than average sources along the Moroccan coast.

The Western Mediterranean results presented here were elaborated on the basis of a pre-existing correlation data set, which was computed using spectral whitening. It is therefore not entirely evident that they can be interpreted in terms of noise source power, although the Atlantic coast is a plausible source region for microseisms. What the results do provide, however, is an image of the 'effective source' of this correlation data set, indicating clearly, for example, that the signal energy in the correlations is not isotropic even after the application of spectral whitening. Further research is needed to assess how spectral whitening affects the source imaging method used here, and related source imaging methods.

\subsection{Case III: microseismic noise at the Swiss Digital Seismic Network}

For the small Swiss data set of $\sim 25$ stations, we analysed correlations for two different months, January and July 2014. The corresponding noise source images for the single- and double-frequency microseismic bands are displayed in Fig. 8. For this data set, we removed windows containing earthquake signals if they were detected by an sta/lta trigger at more than 20 stations.

In the double-frequency band, we observe a two-sided pattern. While the pattern is similar for both January and July, the observed asymmetries are $\sim 50$ per cent stronger in January, that is during the northern winter when storm activity in the North Atlantic is high. Moreover, the directionality of noise sources tips more towards the Mediterranean in summer.

The single-frequency band is distinguished by an even more pronounced seasonal dependence of the noise sources. In January, strong single-frequency noise sources are located west to northwest of the array in the North Atlantic, whereas in July, source power is elevated towards various directions. The source directions found here are broadly consistent with those of Yang \& Ritzwoller (2008), who show the azimuthal dependence of noise correlation signal-to-noise ratios for northern summer and northern winter at a European array.

\subsection{Synthetic test of the imaging procedure}

To understand how well the imaging procedure retrieves an input model, we constructed a synthetic test for the case of the Earth's hum. Compared to their microseism counterparts, the long-period surface waves of the hum propagate over very long distances before being attenuated. As a result, constraining the sources of hum is expected to be challenging, as sensitivity to sources drops off slowly and kernels may 'smear out' the imaged source PSD over large areas. 
January
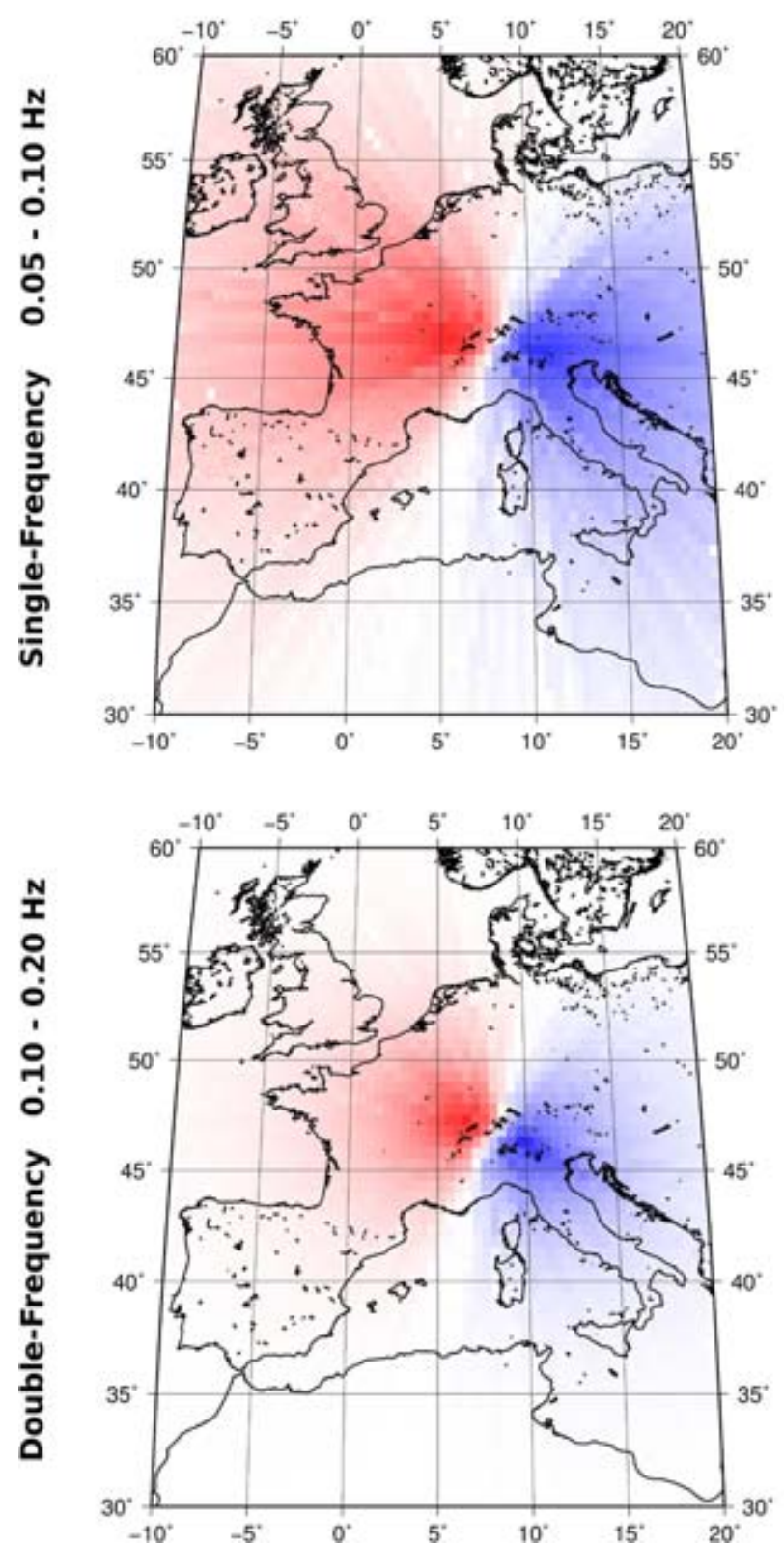

July
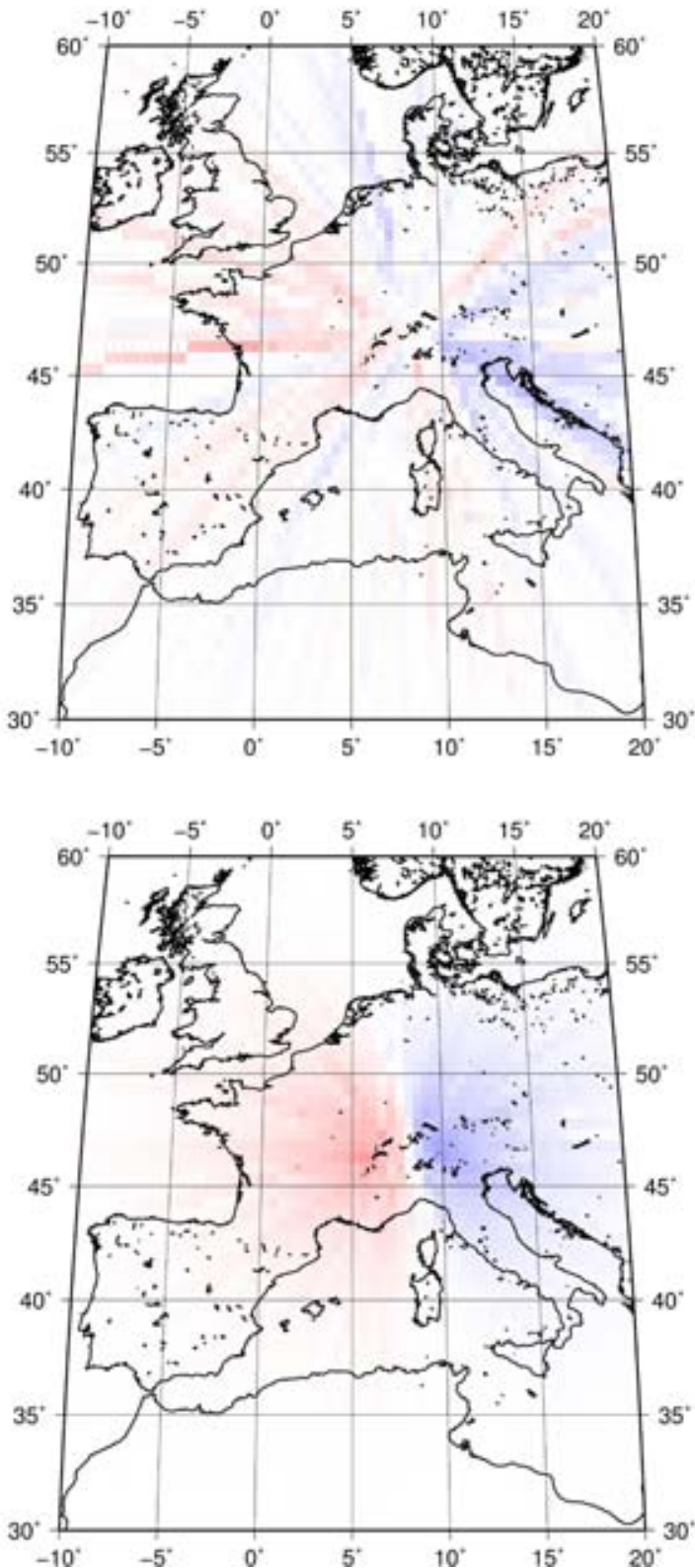

Figure 8. Maximum-normalized power-spectral density distribution for the Swiss Digital Seismic Network. Top: images for the single-frequency band from 0.05 to $0.10 \mathrm{~Hz}$ for January (left) and July 2014 (right). Bottom: as above but for the double-frequency band from 0.10 to $0.20 \mathrm{~Hz}$.

To assess this effect, we use a spherically symmetric Earth model (ak135; Kennett et al. 1995) and constructed synthetic correlations as follows:

We subdivide the Earth's surface into approx. 8000 source locations on a regular grid. We then define a source PSD mask, $S(x)$, that prescribes a location-dependent source PSD factor. We restrict the analysis to a frequency-independent $S(x)$, as our observational analysis treats narrow frequency bands. With this source mask, and under the assumption that sources are spatially uncorrelated, we construct the synthetic correlation function between stations $a, b$ as

$C\left(\mathbf{x}_{a}, \mathbf{x}_{b}\right)=\sum_{n=1}^{N} G\left(\mathbf{y}_{\mathbf{n}}, \mathbf{y}_{\mathbf{a}}\right) G^{*}\left(\mathbf{y}_{\mathbf{n}}, \mathbf{y}_{\mathbf{b}}\right) S\left(\mathbf{y}_{\mathbf{n}}\right) \mathrm{d} \mathbf{y}$. which is a discrete approximation to eq. (5), for vertical components only. $N$ is the number of source locations. Single Green's functions are constructed using vertical point force sources in INSTASEIS (van Driel et al. 2015). We evaluate the synthetics that we constructed in exactly the same way as the observed correlations, except that no signal-to-noise threshold is used. We thus recover an imaged $\tilde{S}(x)$.

A comparison of original $S(x)$ and retrieved images $\tilde{S}(x)$ is shown in Fig. 9. Two cases are presented. Two narrow Gaussian sources, shown by the upper two plots, are placed in the North Atlantic and Indian Ocean, respectively. These are used as an input to the synthetic correlations constructed according to eq. (24). The lower two plots show the retrieved images.

The test demonstrates that the method is, in principle, effective. The narrow sources are imaged broadly across the ocean into which 

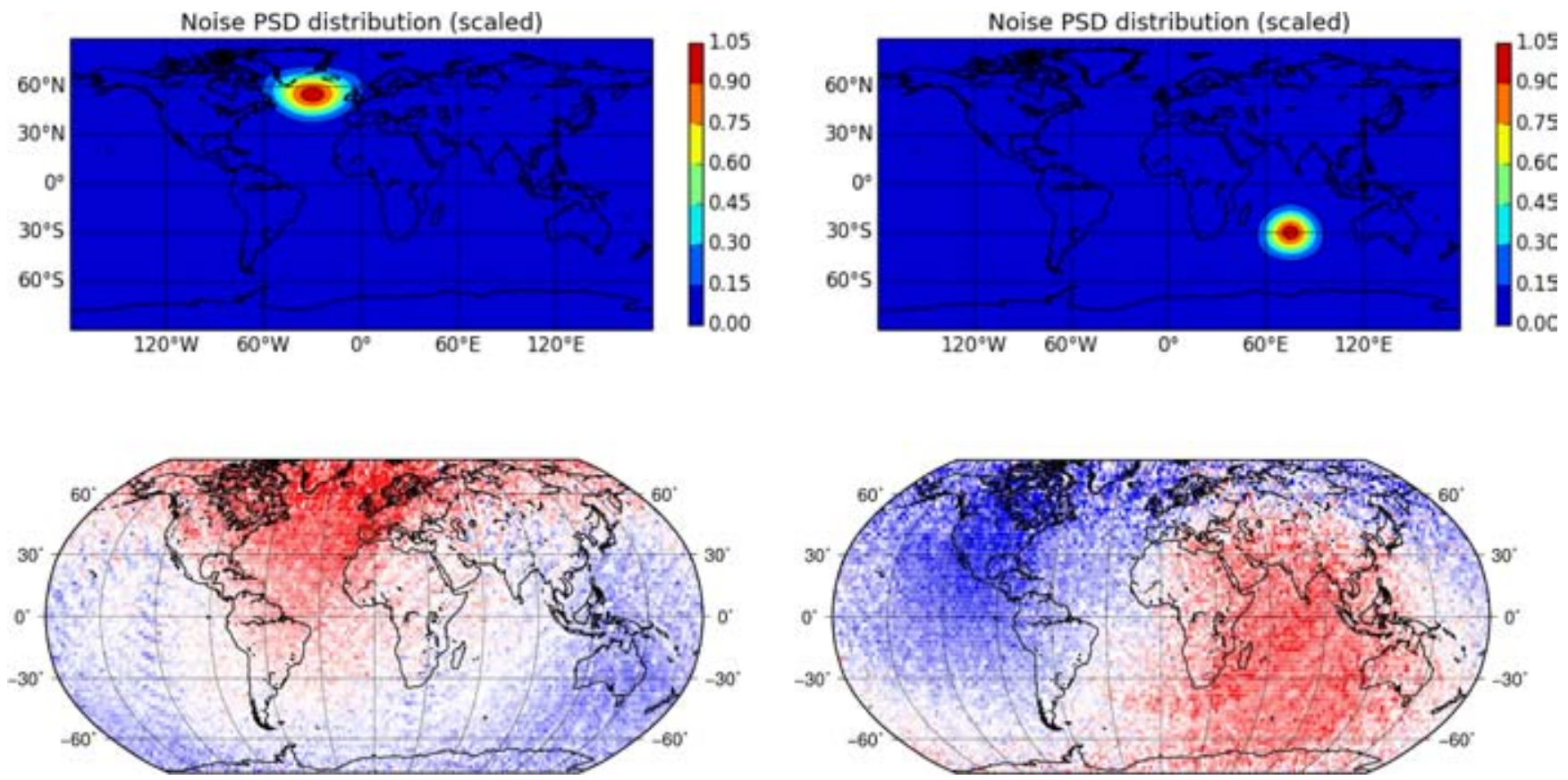

Figure 9. Synthetic test for the retrieval of two Gaussian-shaped source distributions. The upper two panels show the input, the spatial distribution of a source PSD which is assumed to be constant with respect to frequency (supposing that the analysis is restricted to a narrow frequency band, as is the case here). The lower panels show the source maps retrieved from the synthetics by the approach presented here.

they are placed; accuracy is limited, most probably, by the array configuration. While it is desirable to constrain single sources more narrowly than is achieved here, the synthetic approach enables us to evaluate future improvements of the method.

\section{DISCUSSION}

We developed and applied a new method for the imaging of ambient noise sources based on the measurement of cross-correlation asymmetry. The presented method has the advantages that (i) Computational requirements are negligible, and it can be applied directly to existing noise correlation data sets without the need for additional processing. One caveat applies: The method is only strictly valid for linearly processed data. Results such as those from the Western Mediterranean show an effective source distribution after pre-processing. Therefore, interpretations in terms of physical noise sources should be treated very cautiously; further research is needed to quantify the effects of nonlinear processing on waveforms, and consequently on the measurement performed here. (ii) In contrast to beamforming techniques, neither a dense array nor the plane-wave assumption are required. (iii) The chosen measurement is independent of absolute noise correlation amplitudes.

In the next paragraphs, we discuss further details of our method, including the underlying assumptions and limitations, the nature of the imaged noise sources and possible future directions of research. Furthermore, we place our method in the context of the ongoing transition from ray-based to finite-frequency tomography.

\subsection{Assumptions and limitations}

The presented imaging method rests on the assumption that noise sources are spatially uncorrelated in the sense of eq. (7). This is a crucial assumption to make large numbers of synthetic correlations computable and to keep the theory tractable. Kimman \&
Trampert (2010) constructed numerical experiments in which noise sources overlapped in time, and thus sources that were not collocated could be correlated. Comparing the resulting correlations to those produced with uncorrelated sources showed that the fundamental mode surface wave converged, given a sufficient number of sources. Convergence was harder to achieve for higher mode surface waves (which are not subject of the analysis here). The result by Kimman \& Trampert (2010) means that correlations are consistent with spatially uncorrelated sources if enough contributions are averaged. Whether an observational stack is long enough to have converged past the effect of spatially correlated sources is hard to judge. For simplicity, we assumed convergence here. To include a criterion for convergence is a possible improvement to the method.

A minor limitation of the method as presented here stems from the simple window selection procedure, where windows are centred on predicted Rayleigh wave group arrivals. This selection may not always match the arriving wave group perfectly, especially in the microseismic range where Rayleigh waves sample complex crustal structure. However, tests with group velocities varying between 2.5 and $3.1 \mathrm{~km} \mathrm{~s}^{-1}$ on the Swiss data set show that the resulting noise source images are not significantly affected, thereby demonstrating the robustness of the method.

In Section 2.3, we proposed to measure logarithmic amplitude ratios between the causal and anticausal parts of the correlation function in order to infer the distribution of noise sources. This measurement is expected to show negligible sensitivity to Earth structure when the reference noise source distribution is isotropic. Strictly speaking, laterally varying Earth structure (in particular, variable attenuation) can exert small effects on the correlation asymmetry even in the presence of isotropically distributed sources (Liu $\&$ Ben-Zion 2013). A rigorous quantification of possible trade-offs is a topic for further research; in the case of a homogeneous reference source distribution, we assume that these effects are small. A full inversion would allow to account for biases induced by Earth structure. For arbitrary noise source distributions, including the first 
update to an initially isotropic distribution, the argument of negligible influence of Earth structure on our measurement does not hold. Once the reference isotropic source distribution is perturbed towards a more realistic one, perturbations to the structural model may affect the asymmetry measurement as well. It follows that an iterative inversion scheme for the noise source distribution should ideally account for 3-D Earth structure in all iterations.

All current methods to image the sources of ambient seismic noise, including the one presented here, implicitly operate under the assumption that the structure of the Earth is known. Since tomographic resolution is finite, this assumption can clearly not be perfectly met even when elaborate Earth models are used to solve the forward problem and compute sensitivity kernels. Consequently, small-scale Earth structure that acts as scatterer does have the potential to act as apparent source of noise. While such trade-offs can never be completely avoided, they may be minimized by future joint inversions for noise sources and Earth structure. The work presented here is intended to contribute to their development.

\subsection{Finite-frequency ambient noise inversions?}

Following the work of Yomogida (1992) and Friederich (1999), seismic inversion for Earth structure started to transition from ray theory to methods based on spatially extended finite-frequency kernels (e.g. Dahlen et al. 2000; Dahlen \& Baig 2002; Yoshizawa \& Kennett 2004, 2005; Zhou et al. 2004; de Vos et al. 2013). These developments appeared when seismic tomography-first applied in the 1970's (Aki \& Lee 1976; Aki et al. 1976; Dziewoński et al. 1977) — had already reached a mature stage.

The inversion for seismic noise sources based on the rigorous computation of sensitivity kernels is emerging and still far from a similar state of maturity. So far, only synthetic inversions have been studied (Hanasoge 2013, 2014), and experience with real data is still very scarce (Basini et al. 2013). Our approach must be seen in this context as a first step in a developing field. It is intended to serve as a data analysis step prior to a complete iterative inversion for noise sources. It should provide insight into the usefulness of a noise correlation data set for the imaging of noise sources, and it should train the physical intuition that is generally needed to produce meaningful solutions of ill-posed inverse problems. In this regard, our imaging of noise sources resembles the ray theory imaging of traveltime delays frequently used to anticipate the outcome of a tomographic traveltime inversion (see, for instance, fig. 2 of Ritsema et al. 2007).

\subsection{Beyond asymmetry}

While we limited our measurements to the easily observable asymmetry of the main surface wave arrivals, additional information on the noise source distribution is certainly contained in other parts of ambient noise correlations. In this context, precursory arrivals that appear prior to the $P$-wave may be particularly valuable (Landès et al. 2010). The study of such signals is, however, beyond the scope of this work. One challenge in analysing such signals is that it is not immediately clear which type of coherent wave gives rise to the correlation here: It may be surface waves incident at a high angle to the receiver-receiver line, or highly coherent body waves incident subvertically at an array, in which case the kernels turn 3-D. Distinguishing the two cases requires analysing several correlations from an array jointly. Investigating into such signals may be a promising future direction of research.

\section{CONCLUSIONS}

We present a method for the imaging of ambient noise sources based on measurements of cross-correlation asymmetry and the computation of ray-theoretical sensitivity kernels. Advantages of the method include the following: (i) The measurement is robust and independent of absolute noise correlation amplitudes that are affected by attenuation and elastic focussing. (ii) Neither a dense array nor a plane-wave assumption are needed. (iii) The method properly accounts for viscoelastic attenuation and images an actual physical quantity, that is, the normalized PSD of noise sources as a function of space and frequency. (iv) Furthermore, the imaging technique operates directly on noise correlation data sets. No additional processing is required, which makes the method fast and computationally inexpensive. The method may serve as a tool to image ambient noise sources on correlation data sets carefully elaborated without nonlinear processing steps and may serve as a tool to image the 'effective source distribution' seen by correlation data sets that were processed using, for example, spectral whitening or one-bit normalization.

Applied to globally recoded hum in the period range from 4 to $20 \mathrm{mHz}$, our method indicates sources that are stronger in the oceans than on the continents, in accord with previous studies. However, well-covered exceptions appear in continental East Asia and Australia (stronger sources), and in the Atlantic Ocean (weaker sources). For microseismic noise recorded at a regional-scale array in Switzerland, our method reveals almost unidirectional noise propagation except for primary microseismic noise in the summer months. The dominant directions are thus functions of both frequency and time.

Our method is intended as a step towards joint inversions for 3-D Earth structure and ambient noise sources. It will serve, in this context, as a fast tool to assess the information on noise sources contained in ambient noise correlations - and thus to gain the physical intuition needed to solve any ill-posed inverse problem. An extension to finite-frequency kernels and other measurements is a topic of further research.

\section{ACKNOWLEDGEMENTS}

The authors wish to express their gratitude towards Laurent Stehly and an anonymous reviewer for their constructive comments that have contributed much to the quality of the manuscript. We would like to thank Robert Clayton, Anne Obermann, Shravan Hanasoge, Martin Schimmel, Éléonore Stutzmann, Michel Campillo and Victor Tsai for useful discussions and brainstorming sessions. Many thanks also go to Korbinian Sager and Evan Delaney for constructive comments. Figures in this paper were elaborated using GMT (Wessel et al. 2013). Processing strongly relies on Obspy (Beyreuther et al. 2010). Synthetic tests were performed using instaseis (van Driel et al. 2015); we thank Martin van Driel for providing the necessary axisem database. This research was supported by the Swiss National Supercomputing Center (CSCS) in the form of the GeoScale and $\mathrm{CH} 1$ projects, by the Swiss National Science Foundation (SNF) under grant 200021_149143 and by the Netherlands Organisation for Scientific Research (VIDI grant 864.11.008). IberArray data are a contribution of the Team Consolider-Ingenio 2010 TOPO-IBERIA (CSD2006-00041).

\section{REFERENCES}

Agnew, D.C. \& Berger, J., 1978. Vertical seismic noise at very low frequencies, J. geophys. Res., 83, 5420-5424. 
Aki, K. \& Lee, W.H.K., 1976. Determination of three-dimensional velocity anomalies under a seismic array using first $\mathrm{P}$ arrival times from local earthquakes - 1. A homogeneous initial model, J. geophys. Res., 81, 4381-4399.

Aki, K. \& Richards, P., 2002. Quantitative Seismology, University Science Books.

Aki, K., Christoffersson, A. \& Husebye, E.S., 1976. Determination of threedimensional seismic structure of the lithosphere, J. geophys. Res., 81, 277-296.

Ardhuin, F., Stutzmann, E., Schimmel, M. \& Mangeney, A., 2011. Ocean wave sources of seismic noise, J. geophys. Res., 116, doi:10.1029/2011JC006952.

Ardhuin, F., Gualtieri, L. \& Stutzmann, E., 2015. How ocean waves rock the earth: Two mechanisms explain microseisms with periods 3 to $300 \mathrm{~s}$, Geophys. Res. Lett., 42(3), 765-772.

Basini, P., Nissen-Meyer, T., Boschi, L., Casarotti, E., Verbeke, J., Schenk, O. \& Giardini, D., 2013. The influence of nonuniform ambient noise on crustal tomography in Europe, Geochem. Geophys. Geosyst., 14(5), 1471-1492.

Bensen, G.D., Ritzwoller, M.H., Barmin, M.P., Levshin, A.L., Lin, F., Moschetti, M.P., Shapiro, N.M. \& Yang, Y., 2007. Processing seismic ambient noise data to obtain reliable broad-band surface wave dispersion measurements, Geophys. J. Int., 169, 1239-1260.

Beyreuther, M., Barsch, R., Krischer, L. \& Wassermann, J., 2010. ObsPy: A Python toolbox for seismology, Seismol. Res. Lett., 81, 47-58.

Boué, P., Poli, P., Campillo, M. \& Roux, P., 2014. Reverberations, coda waves and ambient noise: correlations at the global scale and retrieval of the deep phases, Earth planet. Sci. Lett., 391, 137-145.

Bromirski, P.D., 2001. Vibrations from the Perfect Storm, Geochem. Geophys. Geosyst., 2(7), doi:10.1029/2000GC000119.

Bromirski, P.D. \& Gerstoft, P., 2009. Dominant source regions of the Earth's "hum" are coastal, Geophys. Res. Lett., 36(13), L13303, doi:10.1029/2009GL038903.

Chevrot, S., Sylvander, M., Benahmed, S., Ponsolles, C., Lefvre, J.M. \& Paradis, D., 2007. Source locations of secondary microseisms in western Europe: evidence for both coastal and pelagic sources, J. geophys. Res., 112(B11), doi:10.1029/2007JB005059.

Chevrot, S. et al., 2014. High-resolution imaging of the Pyrenees and Massif Central from the data of the PYROPE and IBERARRAY portable array deployments, J. Geophys. Res., 119(8), 6399-6420.

Cupillard, P. \& Capdeville, Y., 2010. On the amplitude of surface waves obtained by noise correlation and the capability to recover the attenuation: a numerical approach, Geophys. J. Int., 181(3), 16871700.

Dahlen, F., Hung, S.-H. \& Nolet, G., 2000. Fréchet kernels for finitefrequency traveltimes - I. Theory, Geophys. J. Int., 141, 157174.

Dahlen, F.A. \& Baig, F.A., 2002. Fréchet kernels for body wave amplitudes, Geophys. J. Int., 150, 440-466.

de Vos, D., Paulssen, H. \& Fichtner, A., 2013. Finite-frequency sensitivity kernels fro two-station surface wave measurements, Geophys. J. Int., 194, 1042-1049.

Delaney, E., 2015. Quantifying biases in ambient noise tomography, MSc Thesis, ETH Zurich.

Díaz, J. et al., 2009. The IBERARRAY broadband seismic network: a new tool to investigate the deep structure beneath Iberia, ORFEUS Newsl., 8, $1-6$.

Dziewoński, A.M., Hager, B.H. \& O’Connell, R.J., 1977. Large-scale heterogeneities in the lower mantle, J. geophys. Res., 82, 239-255.

Essen, H.-H., Krüger, F., Dahm, T. \& Grevemeyer, I., 2003. On the generation of secondary microseisms observed in northern and central Europe, J. geophys. Res., 108(B10), doi:10.1029/2002JB002338.

Fichtner, A., 2014. Source and processing effects on noise correlations, Geophys. J. Int., 197, 1527-1531.

Fichtner, A., 2015. Source-structure trade-offs in ambient noise correlations, Geophys. J. Int., 202(1), 678-694.

Fichtner, A., Bunge, H.-P. \& Igel, H., 2006. The adjoint method in seismology-I. Theory, Phys. Earth planet. Inter., 157, 86-104.
Friederich, W., 1999. Propagation of seismic shear and surface waves in a laterally heterogeneous mantle by multiple forward scattering, Geophys. J. Int., 136, 180-204.

Froment, B., Campillo, M., Roux, P., Gouédard, P., Verdel, A. \& Weaver, R.L., 2010. Estimation of the effect of nonisotropically distributed energy on the apparent arrival time in correlations, Geophysics, 75, SA85-SA93.

Fukao, Y., Nishida, K. \& Kobayashi, N., 2010. Seafloor topography, ocean infragravity waves, and background Love and Rayleigh waves, J. geophys. Res., 115(B4), doi:10.1029/2009JB006678.

Gal, M., Reading, A., Ellingsen, S., Gualtieri, L., Koper, K., Burlacu, R., Tkalčić, H. \& Hemer, M., 2015. The frequency dependence and locations of short-period microseisms generated in the southern ocean and west pacific, J. geophys. Res., doi:10.1002/2015JB012210.

Groos, J., Bussat, S. \& Ritter, J., 2012. Performance of different processing schemes in seismic noise cross-correlations, Geophys. J. Int., 188(2), 498-512.

Gualtieri, L., Stutzmann, E., Capdeville, Y., Ardhuin, F., Schimmel, M., Mangeney, A. \& Morelli, A., 2013. Modelling secondary microseismic noise by normal mode summation, Geophys. J. Int., 193(3), 1732-1745.

Hadziioannou, C., Gaebler, P., Schreiber, U., Wassermann, J. \& Igel, H., 2012. Examining ambient noise using co-located measurements of rotational and translational motion, J. Seis., 16, 787-796.

Halliday, D. \& Curtis, A., 2008. Seismic interferometry, surface waves and source distribution, Geophys. J. Int., 175, 1067-1087.

Hanasoge, S.M., 2013. The influence of noise sources on cross-correlation amplitudes, Geophys. J. Int., 192(1), 295-309.

Hanasoge, S.M., 2014. Measurements and kernels for source-structure inversions in noise tomography, Geophys. J. Int., 196(2), 971-985.

Hasselmann, K., 1963. A statistical analysis of the generation of microseisms, Rev. Geophys., 1(2), 177-210.

Kedar, S., Longuet-Higgins, M., Webb, F., Graham, N., Clayton, R. \& Jones, C., 2008. The origin of deep ocean microseisms in the north atlantic ocean, Proc. R. Soc. A, 464, 777-793.

Kennett, B.L.N., Engdahl, E.R. \& Buland, R., 1995. Constraints on seismic velocities in the Earth from traveltimes, Geophys. J. Int., 122, 108-124.

Kimman, W. \& Trampert, J., 2010. Approximations in seismic interferometry and their effects on surface waves, Geophys. J. Int., 182, 461-476.

Kurrle, D. \& Widmer-Schnidrig, R., 2006. Spatiotemporal features of the Earth's background oscillations observed in central Europe, Geophys. Res. Lett., 33(24), L24304, doi:10.1029/2006GL028429.

Kurrle, D. \& Widmer-Schnidrig, R., 2008. The horizontal hum of the Earth: a global background of spheroidal and toroidal modes, Geophys. Res. Lett., 35(6), L06304, doi:10.1029/2007GL033125.

Landès, M., Hubans, F., Shapiro, N.M., Paul, A. \& Campillo, M., 2010. Origin of deep ocean microseisms by using teleseismic body waves, $J$. geophys. Res., 115(B5), doi:10.1029/2009JB006918.

Liu, X. \& Ben-Zion, Y., 2013. Theoretical and numerical results on effects of attenuation on correlation functions of ambient seismic noise, Geophys. J. Int., 194(3), 1966-1983.

Lobkis, O.I. \& Weaver, R.L., 2001. On the emergence of the Green's function in the correlations of a diffuse field, J. acoust. Soc. Am., 110, 3011-3017.

Longuet-Higgins, M.S., 1950. A theory of the origin of microseisms, Phil. Trans. R. Soc. Lond., A, 243(857), 1-35.

Mordret, A., Landès, M., Shapiro, N.M., Singh, S.C., Roux, P. \& Barkved, O.I., 2013. Near-surface study at the Valhall oil field from ambient noise surface wave tomography, Geophys. J. Int., 193(3), 1627-1643.

Nishida, K., 2014. Source spectra of seismic hum, Geophys. J. Int., 199(1), 416-429.

Nishida, K. \& Fukao, Y., 2007. Source distribution of earth's background free oscillations, J. geophys. Res., 112(B6), doi:10.1029/2006JB004720.

Pedersen, H.A. \& Krüger, F., 2007. Influence of the seismic noise characteristics on noise correlations in the baltic shield, Geophys. J. Int., 168(1), 197-210.

Prieto, G.A., Denolle, M., Lawrence, J.F. \& Beroza, G.C., 2011. On amplitude information carried by the ambient seismic field, C. R. Geosci., 343(8), 600-614.

Reading, A.M., Koper, K.D., Gal, M., Graham, L.S., Tkalčić, H. \& Hemer, M.A., 2014. Dominant seismic noise sources in the southern ocean and 
west pacific, 2000-2012, recorded at the Warramunga seismic array, Australia, Geophys. Res. Lett., 41(10), 3455-3463.

Rhie, J. \& Romanowicz, B., 2004. Excitation of Earth's continuous free oscillations by atmosphere-ocean-seafloor coupling, Nature, 431(7008), 552-556.

Rhie, J. \& Romanowicz, B., 2006. A study of the relation between ocean storms and the Earth's hum, Geochem. Geophys. Geosyst., 7(10), doi:10.1029/2006GC001274.

Ritsema, J., McNamara, A.K. \& Bull, A.L., 2007. Tomographic filtering of geodynamic models: implications for model interpretation and large-scale mantle structure, J. geophys. Res., 112, doi:10.1029/2006JB004566.

Rudin, W., 1966. Real and Complex Analysis, McGraw-Hill.

Sabra, K.G., Gerstoft, P., Roux, P. \& Kuperman, W.A., 2005. Surface wave tomography from microseisms in Southern California, Geophys. Res. Lett., 32, doi:10.1029/2005GL023155.

Sánchez-Sesma, F.J. \& Campillo, M., 2006. Retrieval of the Green's function from cross correlation: the canonical elastic problem, Bull. seism. Soc. Am., 96, 1182-1191.

Schimmel, M., 1999. Phase cross-correlations: Design, comparisons, and applications, Bull. seism. Soc. Am., 89(5), 1366-1378.

Schimmel, M., Stutzmann, E. \& Gallart, J., 2011. Using instantaneous phase coherence for signal extraction from ambient noise data at a local to a global scale, Geophys. J. Int., 184(1), 494-506.

Schulte-Pelkum, V., Earle, P.S. \& Vernon, F.L., 2004. Strong directivity of ocean-generated seismic noise, Geochem. Geophys. Geosyst., 5(3), doi:10.1029/2003GC000520.

Sergeant, A., Stutzmann, E., Maggi, A., Schimmel, M., Ardhuin, F. \& Obrebski, M., 2013. Frequency-dependent noise sources in the North Atlantic Ocean, Geochem. Geophys. Geosyst., 14(12), 5341-5353.

Shapiro, N.M., Campillo, M., Stehly, L. \& Ritzwoller, M., 2005. High resolution surface wave tomography from ambient seismic noise, Science, 307, 1615-1618.

Shen, Y., Ren, Y., Gao, H. \& Savage, B., 2012. An improved method to extract very-broadband empirical greens functions from ambient seismic noise, Bull. seism. Soc. Am., 102(4), 1872-1877.

Stehly, L., Campillo, M. \& Shapiro, N.M., 2006. A study of the seismic noise from its long-range correlation properties, J. geophys. Res., 111(B10), B10306, doi:10.1029/2005JB004237.

Stehly, L., Fry, B., Campillo, M., Shapiro, N., Guilbert, J., Boschi, L. \& Giardini, D., 2009. Tomography of the alpine region from observations of seismic ambient noise, Geophys. J. Int., 178(1), 338-350.

Stutzmann, E., Ardhuin, F., Schimmel, M., Mangeney, a. \& Patau, G., 2012. Modelling long-term seismic noise in various environments, Geophys. J. Int., 191(2), 707-722.

Tarantola, A., 1988. Theoretical background for the inversion of seismic waveforms, including elasticity and attenuation, Pure appl. Geophys., 128, 365-399.

Thurner, S., Palomeras, I., Levander, A., Carbonell, R. \& Lee, C.-T., 2014. Ongoing lithospheric removal in the western Mediterranean: evidence from Ps receiver functions and thermobarometry of Neogene basalts (PICASSO project), Geochem. Geophys. Geosyst., 15(4), 11131127.

Tian, Y. \& Ritzwoller, M.H., 2015. Directionality of ambient noise on the Juan de Fuca plate: implications for source locations of the primary and secondary microseisms, Geophys. J. Int., 201(1), 429-443.

Traer, J. \& Gerstoft, P., 2014. A unified theory of microseisms and hum, $J$. geophys. Res., 119(4), 3317-3339.

Traer, J., Gerstoft, P., Bromirski, P.D. \& Shearer, P.M., 2012. Microseisms and hum from ocean surface gravity waves, J. Geophys. Res., 117(B11), B11307, doi:10.1029/2012JB009550.

Tromp, J., Tape, C. \& Liu, Q., 2005. Seismic tomography, adjoint methods, time reversal and banana-doughnut kernels, Geophys. J. Int., 160, 195216.

Tromp, J., Luo, Y., Hanasoge, S. \& Peter, D., 2010. Noise cross-correlation sensitivity kernels, Geophys. J. Int., 183, 791-819.

Tsai, V.C., 2009. On establishing the accuracy of noise tomography traveltime measurements in a realistic medium, Geophys. J. Int., 178, 15551564 . van Driel, M., Krischer, L., Stähler, S.C., Hosseini, K. \& Nissen-Meyer, T., 2015. Instaseis: instant global seismograms based on a broadband waveform database, Solid Earth, 6(2), 701-717.

Verbeke, J., Boschi, L., Stehly, L., Kissling, E. \& Michelini, A., 2012. Highresolution rayleigh-wave velocity maps of central Europe from a dense ambient-noise data set, Geophys. J. Int., 188(3), 1173-1187.

Wapenaar, K., 2004. Retrieving the elastodynamic Green's function of an arbitrary inhomogeneous medium by cross correlation, Phys. Rev. Lett., 93, doi:10.1103/PhysRevLett.93.254301.

Wapenaar, K. \& Fokkema, J., 2006. Green's function representations for seismic interferometry, Geophysics, 71, S133-S146.

Weaver, R.L. \& Lobkis, O.I., 2004. Diffuse fields in open systems and the emergence of Green's function, J. acoust. Soc. Am., 116, 2731-2734.

Wessel, P., Smith, W. H.F., Scharroo, R., Luis, J. \& Wobbe, F., 2013. Generic mapping tools: improved version released, EOS, Trans. Am. geophys. Un., 94(45), 409-410.

Woodard, M.F., 1997. Implications of localized, acoustic absorption for heliotomographic analysis of sunspots, Astrophys. J., 485, 890-894.

Yang, Y. \& Ritzwoller, M.H., 2008. Characteristics of ambient seismic noise as a source for surface wave tomography, Geochem. Geophys. Geosyst., 9(2), doi:10.1029/2007GC001814.

Yao, H. \& Van Der Hilst, R.D., 2009. Analysis of ambient noise energy distribution and phase velocity bias in ambient noise tomography, with application to se tibet, Geophys. J. Int., 179(2), 1113-1132.

Yomogida, K., 1992. Fresnel zone inversion for lateral heterogeneities in the Earth, Pure appl. Geophys., 138, 391-406.

Yoshizawa, K. \& Kennett, B.L.N., 2004. Multi-mode surface wave tomography for the Australian region using a 3-stage approach incorporating finite-frequency effects, J. geophys. Res., 109, doi: 10.1029/2002JB002254.

Yoshizawa, K. \& Kennett, B.L.N., 2005. Sensitivity kernels for finitefrequency surface waves, Geophys. J. Int., 162, 910-926.

Zhou, Y., Dahlen, F.A. \& Nolet, G., 2004. Three-dimensional sensitivity kernels for surface wave observables, Geophys. J. Int., 158, 142-168.

\section{SUPPORTING INFORMATION}

Additional Supporting Information may be found in the online version of this paper:

Figure S1. We argue that the strongest coherence of earthquake signals is achieved on relatively small arrays. The smallest arrays of STS-1 seismometers in our data set are the Berkeley Digital Seismic Network and the Southern California Seismic Network. Excluding these arrays (right map) decreases the resolution of the resulting map, but the global pattern does not change.

Figure S2. The second argument relies on a comparison to the phase cross-correlation. This amplitude-independent correlation function emphasizes weak, coherent signals. Again, no large-scale differences between the processing we used (left map) and the phase cross-correlation, which we used for testing purposes, can be observed (http://gji.oxfordjournals.org/lookup/ suppl/doi:10.1093/gji/ggv460/-/DC1).

Please note: Oxford University Press is not responsible for the content or functionality of any supporting materials supplied by the authors. Any queries (other than missing material) should be directed to the corresponding author for the paper.

\section{APPENDIX A: AMPLITUDE MEASUREMENTS}

\section{A1 Representation of the misfit variation}

To first demonstrate the statement from eq. (6) concerning the general representation of a misfit variation $\delta \chi$ in terms of a variation in the correlation function $\delta C$ and an adjoint source $f$. For this, we 
note that the real-valued misfit functional $\chi$ is a function of the interstation correlation $C_{i j}\left(\mathbf{x}_{1}, \mathbf{x}_{2}, \omega\right)$ :

$\chi=\chi\left[C_{i j}\left(\mathbf{x}_{1}, \mathbf{x}_{2}, \omega\right)\right]$.

The first variation of $\chi$ in the direction of the infinitesimal perturbation $\delta C_{i j}\left(\mathbf{x}_{1}, \mathbf{x}_{2}, \omega\right)$ is a linear functional in $\delta C_{i j}\left(\mathbf{x}_{1}, \mathbf{x}_{2}, \omega\right)$ itself. As a consequence of Riesz' theorem (e.g. Rudin 1966), this functional can be uniquely represented by the scalar product with some function $f(\omega)$, that is, by the integral

$\delta \chi=\int_{-\infty}^{\infty} \delta C_{i j}\left(\mathbf{x}_{1}, \mathbf{x}_{2}, \omega\right) f(\omega) \mathrm{d} \omega$.

The frequency-domain variation $\delta C_{i j}\left(\mathbf{x}_{1}, \mathbf{x}_{2}, \omega\right)$ is the Fourier transform of the real-valued time-domain perturbation $\delta C_{i j}\left(\mathbf{x}_{1}, \mathbf{x}_{2}, t\right)$. Therefore, the following relation holds:

$$
\begin{aligned}
\delta \chi & =\int_{-\infty}^{\infty} \delta C_{i j}^{*}\left(\mathbf{x}_{1}, \mathbf{x}_{2}, \omega\right) f^{*}(\omega) \mathrm{d} \omega \\
& =\int_{-\infty}^{\infty} \delta C_{i j}\left(\mathbf{x}_{1}, \mathbf{x}_{2},-\omega\right) f^{*}(\omega) \mathrm{d} \omega \\
& =\int_{-\infty}^{\infty} \delta C_{i j}\left(\mathbf{x}_{1}, \mathbf{x}_{2}, \omega\right) f^{*}(-\omega) \mathrm{d} \omega
\end{aligned}
$$

We deduce from eq. (A3) that $f(-\omega)=f^{*}(\omega)$, meaning that $f(\omega)$ can be interpreted as the Fourier transform of a real-valued function as well. Taking advantage of this result, we can rewrite eq. (A2) as an integral over positive frequencies only:

$\delta \chi=2 \operatorname{Re} \int_{0}^{\infty} \delta C_{i j}\left(\mathbf{x}_{1}, \mathbf{x}_{2}, \omega\right) f(\omega) \mathrm{d} \omega$.

This is the result from eq. (6). From a physics perspective, the function $f(\omega)$ plays the role of a frequency-domain adjoint source (e.g. Tarantola 1988; Tromp et al. 2005; Fichtner et al. 2006).

\section{A2 Adjoint source for measurements of logarithmic amplitude ratios}

In this paragraph, we derive the adjoint source for calculating sensitivity kernels. This derivation is specific for the chosen measurement of logarithmic amplitude ratios (eq. 11) and the chosen misfit function (eq. 13). The measurement $A$ and misfit $\chi$ are:

$$
A=\ln \frac{\int_{-\infty}^{\infty}\left[w_{+}(t) C_{i j}(t)\right]^{2} \mathrm{~d} t}{\int_{-\infty}^{\infty}\left[w_{-}(t) C_{i j}(t)\right]^{2} \mathrm{~d} t}=\ln \frac{E_{+}}{E_{-}}
$$

$\chi=\frac{1}{2}\left[A-A^{0}\right]^{2}$

which leads to a misfit variation with respect to the model parameters of

$\delta \chi=\left[A-A^{0}\right] \cdot \delta A=\left[A-A^{0}\right] \cdot\left[\frac{\delta E_{+}}{E_{+}}-\frac{\delta E_{-}}{E_{-}}\right]$.

We now consider the energy in the time window on the causal side of the correlation function,

$E_{+}=\int_{-\infty}^{\infty}\left[w_{+}(t) C(t)\right]^{2} \mathrm{~d} t$

To avoid clutter, we simplified the correlation function from $C_{i j}$ to $C$ and use identical symbols for time- and frequency-domain quantities. The variation of $E_{+}$with respect to $C$ is

$$
\begin{aligned}
\delta E_{+} & =2 \int_{-\infty}^{\infty} w_{+}^{2}(t) C(t) \delta C(t) \mathrm{d} t \\
& =\frac{1}{\pi} \int_{-\infty}^{\infty}\left[w_{+}^{2}(\omega) * C(\omega)\right]^{*} \delta C(\omega) \mathrm{d} \omega,
\end{aligned}
$$

where the second identity follows from Parseval's theorem. The symbol $*$ denotes convolution, and $w_{+}^{2}(\omega)$ is the Fourier transform of the squared time window $w_{+}^{2}(t)$. Similarly, the variation of the energy in the anticausal time window is

$$
\begin{aligned}
\delta E_{-} & =2 \int_{-\infty}^{\infty} w_{-}^{2}(t) C(t) \delta C(t) \mathrm{d} t \\
& =\frac{1}{\pi} \int_{-\infty}^{\infty}\left[w_{-}^{2}(\omega) * C(\omega)\right]^{*} \delta C(\omega) \mathrm{d} \omega,
\end{aligned}
$$

With (A6) and (A7) in mind, we can compute the variation of the asymmetry misfit defined in eq. (13). Substituting eqs (A6) and (A7) into the misfit variation, we obtain

$$
\begin{aligned}
\delta \chi= & 2 \operatorname{Re} \int_{0}^{\infty} \delta C(\omega) f(\omega) \mathrm{d} \omega \\
= & 2 \operatorname{Re} \int_{0}^{\infty} \int_{\oplus}\left[G_{i n}\left(\mathbf{x}_{1}, \mathbf{y}, \omega\right)\right. \\
& \left.\times G_{j m}^{*}\left(\mathbf{x}_{2}, \mathbf{y}, \omega\right) \delta S_{n m}(\mathbf{y}, \omega)\right] f(\omega) \mathrm{d} \omega \mathrm{d} \mathbf{y}
\end{aligned}
$$

with $f(\omega)$ given by

$$
\begin{aligned}
f(\omega)=\left(A-A^{0}\right)\left[\frac{1}{\pi E_{+}}\right. & \left(w_{+}^{2}(\omega) * C(\omega)\right)^{*} \\
& \left.\quad-\frac{1}{\pi E_{-}}\left(w_{-}^{2}(\omega) * C(\omega)\right)^{*}\right] .
\end{aligned}
$$

\section{APPENDIX B: RAY APPROXIMATION}

To derive the decay of the ray-theoretical sensitivity along the great circle through the receiver pair, we let both receivers be positioned on the $x$-axis at equal distances from the origin, as shown in Fig. B1. Furthermore, we work under the assumptions used for the computation of the 2-D examples in Section 2.4. These assumptions include a homogeneous reference distribution of noise sources, a homogeneous unbounded medium, and acoustic wave propagation. In order to preserve the total sensitivity, we ascribe to each $x$-coordinate along the ray the sensitivity integrated along the ray-perpendicular direction, that is,

$K_{\text {ray }}(x)=\int_{-\infty}^{\infty} K(x, y) \mathrm{d} y$.

Recalling eq. (20), the 2-D sensitivity kernel is given by

$K(\mathbf{x})=\operatorname{Re} \frac{1}{4 \pi \omega \rho^{2} v^{3}} \frac{f}{\sqrt{r_{1} r_{2}}} \mathrm{e}^{i \frac{\omega}{v}\left(r_{2}-r_{1}\right)} \mathrm{e}^{-\frac{\omega}{2 v Q}\left(r_{1}+r_{2}\right)}$.

The 2-D kernel $K(\mathbf{x})$ is constant along the hyperbolae $r_{2}-r_{1}=$ const., which are asymptotically close to straight lines crossing the origin. Furthermore, $K(\mathbf{x})$ is an oscillatory function along the $y$-axis for constant $x$. We can obtain an approximation of the integral (B1) by considering integrals over just one oscillation for a fixed $x$ :

$K_{\text {ray }}^{[a, b]}(x)=\int_{a}^{b} K(x, y) \mathrm{d} y$.

Assuming that $x$ is located at sufficient distance from the receiver pair, we observe the following when changing $x$ to $\gamma x$, where $\gamma$ is 


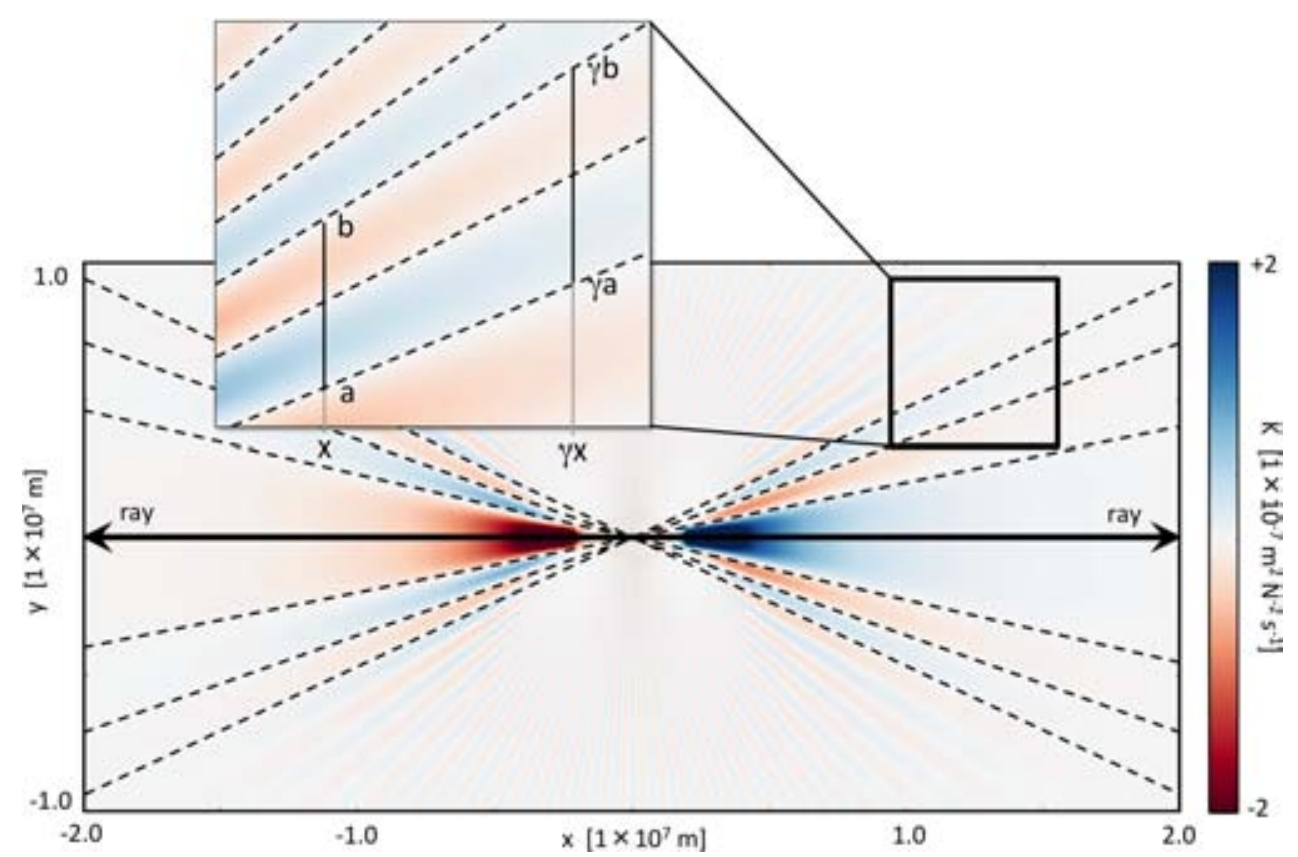

Figure B1. Illustration for the computation of a ray theoretical noise source kernel. In the absence of attenuation, the integral of $K(\mathbf{x})$ over $y \in[a, b]$ is equal to the integral over $y \in[\gamma a, \gamma b]$ because stretching and scaling of one oscillation in the kernel compensate each other.

some real number (see also Fig. B1): (i) The same oscillation is now approximately located within the interval $\gamma a \leq y \leq \gamma b$, meaning that the oscillation period increases by the factor $\gamma$. (ii) The scaling factor $\left(r_{1} r_{2}\right)^{-1 / 2} \approx|\mathbf{x}|^{-1}$ in eq. (B2) changes to $\gamma^{-1}|\mathbf{x}|^{-1}$. (iii) The attenuation term $\frac{\omega}{2 v Q}\left(r_{1}+r_{2}\right) \approx \frac{\omega}{v Q}|\mathbf{x}|$ is modified to $\frac{\gamma \omega}{v Q}|\mathbf{x}|$.

Effects (i) and (ii), that is stretching and scaling of the oscillation, cancel each other exactly; meaning that the ray-perpendicular integral (B1) is constant with respect to $x$ when $Q$ is infinite. Finite $Q$ adds the attenuation factor $\mathrm{e}^{-\frac{\gamma \omega}{v Q}|\mathbf{x}|}$ to the integrand. Since $\mathrm{e}^{-\frac{\gamma \omega}{v Q}|\mathbf{x}|} \leq \mathrm{e}^{-\frac{\gamma \omega}{v Q} x}$, we obtain the following upper bound for the raytheoretical kernel amplitude:

$K_{\text {ray }}(x) \approx$ const. $\mathrm{e}^{-\frac{\omega x}{v Q}}$.
The constant in eq. (B4) absorbs the scaling factors from the 2-D kernel in eq. (B2), as well as the sum of the integrals over all individual oscillations.

The fact that $K_{\text {ray }}(x)$ is constant in the absence of attenuation highlights the global nature of noise propagation. Noise sources at arbitrary distance from the receiver pair can have the same effect as noise sources close to the receivers. Only the presence of attenuation reduces the impact of distant noise sources. In contrast to the amplitudes of travelling waves that decay as $\mathrm{e}^{-\frac{\omega}{2 v Q} x}$, the amplitude of the noise source kernel decays more quickly as $\mathrm{e}^{-\frac{\gamma \omega}{v Q} x}$.

We finally note that our analysis makes no assumptions on the properties of the adjoint source $f$. It is therefore valid for all types of measurements. 